\title{
Constraints of Asymptotic Character and Attainability Problems
}

\author{
Alexander G. Chentsov ${ }^{1,2}$ \\ ${ }^{1}$ Institute of Mathematics and Mechanics Named after N.N. Krasovskii, Ural Branch of the Russian Academy of Sciences, \\ Yekaterinburg, Russia \\ ${ }^{2}$ Institute of Radioelectronics and Information Technologies, Ural Federal University Named after the First President of \\ Russia B.N. Yeltsin, Yekaterinburg, Russia \\ Email: chentsov@imm.uran.ru
}

How to cite this paper: Chentsov, A.G. (2017) Constraints of Asymptotic Character and Attainability Problems. Intelligent Information Management, 9, 206-228. https://doi.org/10.4236/iim.2017.95011

Received: July 25, 2017

Accepted: September 16, 2017

Published: September 19, 2017

Copyright (C) 2017 by author and Scientific Research Publishing Inc. This work is licensed under the Creative Commons Attribution International License (CC BY 4.0).

http://creativecommons.org/licenses/by/4.0/ (c) (i) Open Access

\begin{abstract}
The attainability problem with "asymptotic constraints" is considered. Concrete variants of this problem arise in control theory. Namely, we can consider the problem about construction and investigation of attainability domain under perturbation of traditional constraints (boundary and immediate conditions; phase constraints). The natural asymptotic analog of the usual attainability domain is attraction set, for representation of which, the Warga generalized controls can be applied. More exactly, for this, attainability domain in the class of generalized controls is constructed. This approach is similar to methods for optimal control theory (we keep in mind approximate and generalized controls of J. Warga). But, in the case of attainability problem, essential difficulties arise. Namely, here it should be constructed whole set of limits corresponding to different variants of all more precise realization of usual solutions in the sense of constraints validity. Moreover, typically, the abovementioned control problems are infinite-dimensional. Real possibility for investigation of the arising limit sets is connected with extension of control space. For control problems with geometric constraints on the choice of programmed controls, procedure of this extensions was realized (for extremal problems) by J. Warga. More complicated situation arises in theory of impulse control. It is useful to note that, for investigation of the problem about constraints validity, it is natural to apply asymptotic approach realized in part of perturbation of standard constraints. And what is more, we can essentially generalize self notion of constraints: namely, we can consider arbitrary systems of conditions defined in terms of nonempty families of sets in the space of usual controls. Thus, constraints of asymptotic character arise.
\end{abstract}

\section{Keywords}

Attraction Set, Topological Space, Ultrafilter 


\section{Informative Discussion of Problem}

We consider general attainability problem in a topological space (TS). For this, we fix a nonempty set $E$ elements of which play the role of usual solutions (sometimes, it is logical to consider elements of $E$ as controls). Moreover, we fix a TS $(\mathbf{H}, \mathbf{t})$ and a mapping $\mathbf{h}: E \rightarrow \mathbf{H}$ called the goal operator. If $e \in E$, then $\mathbf{h}(e) \in \mathbf{H}$ is the concrete result of the choice of $e$. If a subset $E_{0}$ of $E$ is given, then image $\mathbf{h}^{1}\left(E_{0}\right)=\left\{\mathbf{h}(e): e \in E_{0}\right\}$ of $E_{0}$ can be considered as attainable set or analog of attainability domain in control theory (see [1] [2]). The closure $\overline{\mathbf{h}^{1}\left(E_{0}\right)}=\operatorname{cl}\left(\mathbf{h}^{1}\left(E_{0}\right), \mathbf{t}\right)$ of $\mathbf{h}^{1}\left(E_{0}\right)$ is a natural generalization: we assume realization of points of $\mathbf{H}$ "in a limit" under precise validity of constraints connected with the set $E_{0}$. In investigation of J. Warga, for optimization problems, the approach using weakening of $E_{0}$-constraints was proposed (see ch. III,IV of monograph [2]). Of course, this approach assumes the natural spreading on attainability problems. Namely, we replace $E_{0}$ by a nonempty family $\mathcal{E}$ of subsets of $E$ with the property

$$
E_{0}=\bigcap_{\Sigma \in \mathcal{E}} \Sigma
$$

(usually, the family $\mathcal{E}$ is directed in the following traditional sense

$$
\forall \Sigma_{1} \in \mathcal{E} \forall \Sigma_{2} \in \mathcal{E} \exists \Sigma_{3} \in \mathcal{E}: \Sigma_{3} \subset \Sigma_{1} \cap \Sigma_{2}
$$

but, now we not discuss this supposition). It is logical to suppose that sets of the family $\mathcal{E}$ are "near" to $E_{0}$ (we keep in mind Warga approach). For every set $\Sigma \in \mathcal{E}$, we consider the set-image $\mathbf{h}^{1}(\Sigma)$ and its closure $\overline{\mathbf{h}^{1}(\Sigma)}$ in TS $(\mathbf{H}, \mathbf{t})$. Later, we can consider the intersection of all sets $\overline{\mathbf{h}^{1}(\Sigma)}, \Sigma \in \mathcal{E}$ instead of $\overline{\mathbf{h}^{1}\left(E_{0}\right)}$; in this representation, the validity of (1.2) is essential. The given intersection is interpreted as attraction set (AS). This construction can be considered as natural analog of the Warga approach for extremal problems (see ch. III, IV in [2]). But, for abstract attainability problem, the above-mentioned construction with employment of AS can be extended very essentially.

Indeed, we can investigate the setting for which the family $\mathcal{E}$ is primarily (it is supposed that the set $E_{0}$ is not given). This family consists of subsets of $E$; usually it is sufficient to suppose that $\mathcal{E}$ is directed (see (1.2)). But, now we not require validity of this condition.

So, let $\mathcal{E}$ be a nonempty family of subsets of $E$. At first, we consider questions connected with the choice of mappings with values in $E$. In particular, we can consider the choice of sequences in $E$. But, sometimes, our possibilities are extended in the case when the choice of nets is admissible. Therefore, now we discuss the choice of nets $\left(x_{\alpha}\right)$ in the set $E$. We consider such nets as asymptotic regimes. Respectively, in $\mathbf{H}$, nets $\left(\mathbf{h}\left(x_{\alpha}\right)\right)$ are realized. If, for a net $\left(x_{\alpha}\right)$ in $E$, under every $\Sigma \in \mathcal{E}$, the condition $x_{\alpha} \in \Sigma$ is fulfilled starting from a certain index, then we consider $\left(x_{\alpha}\right)$ as admissible asymptotic regime. The following question arises: for which $y \in \mathbf{H}$, an admissible asymptotic regime $\left(x_{\alpha}\right)$ with the property of convergence of $(\mathbf{h}(x(\alpha)))$ to $y$ can be chosen. Such 
points $y$ are interpreted as attainable elements of $\mathbf{H}$. We consider the problem about construction of the set of all attainable elements. We call his AS. In addition, under condition (1.2) our new definition of AS is equivalent to previously mentioned definition (see formula (3.3.10) in [3]). If (1.2) is not fufilled, then we can to replace $\mathcal{E}$ on the family $\mathcal{E}_{\mathrm{f}}$ of all finite intersections of sets of $\mathcal{E}$. Then AS defined by the convergence of nets (that is, by asymptotic regimes) coincides with intersection of all sets $\overline{\mathbf{h}^{1}(\Sigma)}, \Sigma \in \mathcal{E}_{\mathbf{f}}$. We note that filters can be used instead of nets (see [4]).

\section{General Notions and Definitions}

We exploit standard set-theoretical symbolics (quantors, propositional connectives; $\varnothing$ is empty set). In the following, $\triangleq$ is equality by definition; $\exists$ ! replaces phrase "exists and uniquely". We take axiom of choice. For every object $x$, by $\{x\}$ we denote singleton containing $x$ (so, $x \in\{x\}$ ). The set is called a family in the case when every element of this set is a set also.

If $H$ is a set, then by $\mathcal{P}(H)$ and $\mathcal{P}^{\prime}(H)$ we denote the families of all and all nonempty subsets of $H$ respectively; so, $\mathcal{P}^{\prime}(H)=\mathcal{P}(H) \backslash\{\varnothing\}$. By $\operatorname{Fin}(H)$ we denote the family of all finite sets of $\mathcal{P}^{\prime}(H)$; so, $\operatorname{Fin}(H)$ is the family of all nonempty finite subsets of $H$. If $\mathcal{H} \in \mathcal{P}^{\prime}(\mathcal{P}(H))$, then $\mathcal{H}$ is a nonempty subfamily of $\mathcal{P}(H)$. Then, for a fixed set $\mathbb{H}$ and a family $\mathcal{H} \in \mathcal{P}^{\prime}(\mathcal{P}(\mathbb{H}))$, we introduce the family

$$
\mathbf{C}_{\mathbb{H}}[\mathcal{H}] \triangleq\{\mathbb{H} \backslash H: H \in \mathcal{H}\} \in \mathcal{P}^{\prime}(\mathcal{P}(\mathbb{H}))
$$

dual with respect to $\mathcal{H}$ (of course, a topology of $\mathbb{H}$ can be used as $\mathcal{H}$; in this case, the family of closed subsets of $\mathbb{H}$ is realized as $\mathbf{C}_{\mathbb{H}}[\mathcal{H}]$ ). It is obviously that

$$
\mathbf{C}_{\mathbb{H}}\left[\mathbf{C}_{\mathbb{H}}[\mathfrak{A}]\right]=\mathfrak{A} \quad \forall \mathfrak{A} \in \mathcal{P}^{\prime}(\mathcal{P}(\mathbb{H}))
$$

Now, we consider other operations with families. So, for every nonempty family $\mathcal{X}$ and a set $Y$,

$$
\left.\mathcal{X}\right|_{Y} \triangleq\{X \cap Y: X \in \mathcal{X}\} \in \mathcal{P}^{\prime}(\mathcal{P}(Y))
$$

is trace of $\mathcal{X}$ on the set $Y$. If $\mathcal{H}$ is a nonempty family, then we suppose that

$$
\left(\{\bigcup\}(\mathcal{H}) \triangleq\left\{\bigcup_{H \in \mathfrak{H}} H: \mathfrak{H} \in \mathcal{P}(\mathcal{H})\right\}\right) \&\left(\{\cap\}_{\mathbf{f}}(\mathcal{H}) \triangleq\left\{\bigcap_{H \in \mathcal{K}} H: \mathcal{K} \in \operatorname{Fin}(\mathcal{H})\right\}\right)
$$

in addition, $\mathcal{H} \subset\{\cup\}(\mathcal{H})$ and $\mathcal{H} \subset\{\cap\}_{\mathbf{f}}(\mathcal{H})$. Of course, in (2.1), we obtain two nonempty families of subsets of union of all sets of $\mathcal{H}$.

For every sets $A$ and $B$, by $B^{A}$ we denote the set of all mappings from $A$ into $B$; of course, under $f \in B^{A}$ and $a \in A, f(a) \in B$ is the $f$-image of the point $a$. For every sets $U$ and $V$, a mapping $g \in V^{U}$, and a set $W \in \mathcal{P}(U)$, by $(g \mid W)$ we denote the contraction of $g$ on the set $W\left((g \mid W) \in V^{W}\right.$ and $(g \mid W)(w) \triangleq g(w) \forall w \in W)$ and $g^{1}(W) \triangleq\{g(x): x \in W\} \in \mathcal{P}(V)$ (the image of $W$ under operation by $g)$. 
Special families. In the following, we consider families of subsets of that or different fixed set. Therefore, in this item, we fix a nonempty set $I$ and consider the family $\mathcal{P}^{\prime}(\mathcal{P}(I))$ of all nonempty subfamilies of $\mathcal{P}(I)$. Among all such subfamilies, we select $\pi$-systems (see p. 14 in monograph [5]) with "zero" and "unit"; then,

$$
\pi[I] \triangleq\left\{\mathcal{I} \in \mathcal{P}^{\prime}(\mathcal{P}(I)) \mid(\varnothing \in \mathcal{I}) \&(I \in \mathcal{I}) \&(A \cap B \in \mathcal{I} \forall A \in \mathcal{I} \forall B \in \mathcal{I})\right\}
$$

is the family of all such $\pi$-systems. Of course, every algebra of subsets of $I$ and every topology on $I$ are $\pi$-systems of the family (2.2); therefore, we introduce

$$
\begin{aligned}
& \text { (alg) }[I] \triangleq\{\mathcal{L} \in \pi[I] \mid I \backslash L \in \mathcal{L} \forall L \in \mathcal{L}\}, \\
& \text { (top) }[I] \triangleq\left\{\tau \in \pi[I] \mid \bigcup_{G \in \mathcal{G}} G \in \tau \forall \mathcal{G} \in \mathcal{P}^{\prime}(\tau)\right\}
\end{aligned}
$$

Every semialgebra of subsets of $I$ (semiring with "unit") is a $\pi$-system of (2.2) also. In the form of

$$
\tilde{\pi}^{0}[I] \triangleq\{\mathcal{I} \in \pi[I] \mid \forall L \in \mathcal{I} \forall x \in I \backslash L \exists \Lambda \in \mathcal{I}:(x \in \Lambda) \&(\Lambda \cap L=\varnothing)\}
$$

we obtain the family of all separable $\pi$-systems of (2.2). Particular case of $\pi$ system of the family (2.3) is realized for $\pi$-systems with singletons:

$$
\pi_{0}^{\prime}[I] \triangleq\{\mathcal{I} \in \pi[I]\{x\} \in \mathcal{I} \forall x \in I\} \in \mathcal{P}^{\prime}\left(\tilde{\pi}^{0}[I]\right)
$$

(in (2.4), we exploit the obvious property $\left.\mathcal{P}(I) \in \pi_{0}^{\prime}[I]\right)$. In the form of

$$
(\mathrm{LAT})_{0}[I] \triangleq\{\mathcal{I} \in \pi[I] \mid A \cup B \in \mathcal{I} \forall A \in \mathcal{I} \forall B \in \mathcal{I}\}
$$

we obtain the family of all lattices with "zero" and "unit". Of course, (alg) $[I] \subset(\mathrm{LAT})_{0}[I]$ and (top) $[I] \subset(\mathrm{LAT})_{0}[I]$. We suppose that

$$
\beta[I] \triangleq\left\{\mathcal{B} \in \mathcal{P}^{\prime}(\mathcal{P}(I)) \mid \forall B_{1} \in \mathcal{B} \forall B_{2} \in \mathcal{B} \exists B_{3} \in \mathcal{B}: B_{3} \subset B_{1} \cap B_{2}\right\}
$$

(the family of all nonempty directed subfamilies of $\mathcal{P}(I)$ ); then, elements of the family

$$
\begin{aligned}
\beta_{0}[I] & \triangleq\{\mathcal{B} \in \beta[I] \mid \varnothing \notin \mathcal{B}\} \\
& =\left\{\mathcal{B} \in \mathcal{P}^{\prime}\left(\mathcal{P}^{\prime}(I)\right) \mid \forall B_{1} \in \mathcal{B} \forall B_{2} \in \mathcal{B} \exists B_{3} \in \mathcal{B}: B_{3} \subset B_{1} \cap B_{2}\right\}
\end{aligned}
$$

are filter bases of the set $I$ and only they. Moreover,

$$
\begin{array}{r}
\mathfrak{F}[I] \triangleq\left\{\mathcal{F} \in \mathcal{P}^{\prime}\left(\mathcal{P}^{\prime}(I)\right) \mid(A \cap B \in \mathcal{F} \forall A \in \mathcal{F} \forall B \in \mathcal{F})\right. \\
\&(\forall F \in \mathcal{F} \forall L \in \mathcal{P}(I)(F \subset L) \Rightarrow(L \in \mathcal{F}))\}
\end{array}
$$

is the family of all filters of $I$ (see ch.I in monograph [6]); elements of the family

$$
\mathfrak{F}_{\mathbf{u}}[I] \triangleq\{\mathcal{U} \in \mathfrak{F}[I] \mid \forall \mathcal{F} \in \mathfrak{F}[I](\mathcal{U} \subset \mathcal{F}) \Rightarrow(\mathcal{U}=\mathcal{F})\}
$$

are ultrafilters of $I$ and only they. If $\mathcal{J} \in \mathcal{P}^{\prime}(\mathcal{P}(I))$, then we suppose that

$$
\mathfrak{F}_{\mathbf{u}}^{0}[I \mid \mathcal{J}] \triangleq\left\{\mathcal{U} \in \mathfrak{F}_{\mathbf{u}}[I] \mid \mathcal{J} \subset \mathcal{U}\right\} .
$$


In addition, the following known (see ch.I in [6]) construction is used: if $\mathcal{B} \in \beta_{0}[I]$, then

$$
(I-\mathbf{f i})[\mathcal{B}] \triangleq\{F \in \mathcal{P}(I) \mid \exists B \in \mathcal{B}: B \subset F\} \in \mathfrak{F}[I]
$$

is the filter of $I$ generated by the base $\mathcal{B}$. Finally, for $x \in I$

$$
(I-\text { ult })[x] \triangleq\{S \in \mathcal{P}(I) \mid x \in S\} \in \mathfrak{F}_{\mathrm{u}}[I]
$$

so, trivial (fixed) ultrafilters are defined. By $(I-\mathrm{ult})[\cdot] \triangleq((I-\mathrm{ult})[x])_{x \in I}$ the natural rule of immersion of $I$ into $\mathfrak{F}_{\mathrm{u}}[I]$ is realized. We note that $(2.6)$ is a nonempty set.

Filters and ultrafilters of $\pi$-systems. Now, we consider "partial" filters and ultrafilters. For this, in the present item, we fix arbitrary $\pi$-system $\mathcal{I} \in \pi[I]$. So, $(I, \mathcal{I})$ is analog of measurable space. In the following, we call $(I, \mathcal{I})$ a meaurable space also; of course, this term is regarded extendedly. We suppose that

$$
(\text { Cen })[\mathcal{I}] \triangleq\left\{\mathcal{Z} \in \mathcal{P}^{\prime}(I) \mid \bigcap_{Z \in \mathcal{K}} Z \neq \varnothing \forall \mathcal{K} \in \operatorname{Fin}(\mathcal{Z})\right\}
$$

Families of (2.8) are nonempty centered subfamilies of $\mathcal{I}$ and only they. Introduce "partial" analogs of filters of (2.5); namely, in

$$
\begin{aligned}
\mathbb{F}^{*}(\mathcal{I}) \triangleq & \left\{\mathcal{F} \in \mathcal{P}^{\prime}(\mathcal{I} \backslash\{\varnothing\}) \mid(A \cap B \in \mathcal{F} \forall A \in \mathcal{F} \forall B \in \mathcal{F})\right. \\
& \&(\forall F \in \mathcal{F} \forall L \in \mathcal{I}(F \subset L) \Rightarrow(L \in \mathcal{F}))\}
\end{aligned}
$$

the set of all $\mathcal{I}$-filters is defined. As a corollary,

$$
\begin{aligned}
\mathbb{F}_{0}^{*}(\mathcal{I}) & \triangleq\left\{\mathcal{U} \in \mathbb{F}^{*}(\mathcal{I}) \mid \forall \mathcal{F} \in \mathbb{F}^{*}(\mathcal{I})(\mathcal{U} \subset \mathcal{F}) \Rightarrow(\mathcal{U}=\mathcal{F})\right\} \\
& =\left\{\mathcal{U} \in \mathbb{F}^{*}(\mathcal{I}) \mid \forall L \in \mathcal{I}(L \cap U \neq \varnothing \forall U \in \mathcal{U}) \Rightarrow(L \in \mathcal{U})\right\} \\
& =\{\mathcal{U} \in(\text { Cen })[\mathcal{C}] \mid \forall \mathcal{C} \in(\text { Cen })[\mathcal{I}](\mathcal{U} \subset \mathcal{C}) \Rightarrow(\mathcal{U}=\mathcal{C})\}
\end{aligned}
$$

is the set of all $\mathcal{I}$-ultrafilters. In connection with (2.10), we note constructions of [7] [8]. Suppose that

$$
\begin{aligned}
\Phi_{\mathcal{I}}(L) & \triangleq\left\{\mathcal{U} \in \mathbb{F}_{0}^{*}(\mathcal{I}) \mid L \in \mathcal{U}\right\} \\
& =\left\{\mathcal{U} \in \mathbb{F}_{0}^{*}(\mathcal{I}) \mid L \cap U \neq \varnothing \forall U \in \mathcal{U}\right\} \forall L \in \mathcal{I}
\end{aligned}
$$

The sets (2.11) play important role in questions of topological equipment of $\mathbb{F}_{0}^{*}(\mathcal{I})$; these questions is considered later. On the other hand,

$$
\mathbb{F}_{0}^{*}(\mathcal{I} \mid \mathcal{J}) \triangleq\left\{\mathcal{U} \in \mathbb{F}_{0}^{*}(\mathcal{I}) \mid \mathcal{J} \subset \mathcal{U}\right\}=\bigcap_{L \in \mathcal{J}} \Phi_{\mathcal{I}}(L) \forall \mathcal{J} \in \mathcal{P}^{\prime}(I)
$$

in (2.12), we obtain the natural analog of (2.7). Now, we consider analogs of (2.11) for which the condition of $\mathcal{I}$-measurability of set defifning (2.11) can be omitted. Namely, let

$$
\mathbf{F}_{0}^{*}[\mathcal{I} \mid A] \triangleq\left\{\mathcal{U} \in \mathbb{F}_{0}^{*}(\mathcal{I}) \mid A \cap U \neq \varnothing \forall U \in \mathcal{U}\right\} \forall A \in \mathcal{P}(I)
$$


Analogously, as some generalization of (2.12), we exploit the following families:

$$
\begin{aligned}
\mathbf{F}_{0}^{*}(\mathcal{I} \mid \mathcal{A}) & \triangleq\left\{\mathcal{U} \in \mathbb{F}_{0}^{*}(\mathcal{I}) \mid A \cap U \neq \varnothing \forall A \in \mathcal{A} \forall U \in \mathcal{U}\right\} \\
& =\bigcap_{A \in \mathcal{A}} \mathbf{F}_{0}^{*}[\mathcal{I} \mid A] \forall \mathcal{I} \in \mathcal{P}^{\prime}(\mathcal{P}(I))
\end{aligned}
$$

Of course, $\Phi_{\mathcal{I}}(L)=\mathbf{F}_{0}^{*}[\mathcal{I} \mid L]$ under $L \in \mathcal{I}$; moreover, $\mathbb{F}_{0}^{*}(\mathcal{I} \mid \mathcal{J})=\mathbf{F}_{0}^{*}(\mathcal{I} \mid \mathcal{J})$ under $\mathcal{J} \in \mathcal{P}^{\prime}(\mathcal{I})$. So, (2.13) is natural generalization of (2.11) and (2.14) is analogous generalization of (2.12). If $x \in I$, then

$$
((I, \mathcal{I})-\text { ult })[x] \triangleq(I-\text { ult })[x] \cap \mathcal{I}=\{L \in \mathcal{I} \mid x \in L\} \in \mathbb{F}^{*}(\mathcal{I})
$$

is considered as trivial $\mathcal{I}$-filter corresponding to point $x$. We recall (see Section 3 in [9]) that

$$
\mathbb{F}_{0, \mathbf{f}}^{*}(\mathcal{I}) \triangleq\left\{\mathcal{U} \in \mathbb{F}_{0}^{*}(\mathcal{I}) \mid \bigcap_{U \in \mathcal{U}} U=\varnothing\right\}=\mathbb{F}_{0}^{*}(\mathcal{I}) \backslash \mathbb{F}_{0, \mathrm{t}}^{*}(\mathcal{I})
$$

where $\mathbb{F}_{0, \mathrm{t}}^{*}(\mathcal{I}) \triangleq\{((I, \mathcal{I})-$ ult $)[x]: x \in I\} \in \mathcal{P}^{\prime}\left(\mathbb{F}^{*}(\mathcal{I})\right)$. In addition (see p.214 in [8]),

$$
\left(\mathcal{I} \in \tilde{\pi}^{0}[I]\right) \Leftrightarrow\left(((I, \mathcal{I})-\text { ult })[x] \in \mathbb{F}_{0}^{*}(\mathcal{I}) \forall x \in I\right)
$$

Remark 2.1. We recall that $\mathcal{P}(I) \in \pi[I]$ (and what is more, $\mathcal{P}(I) \in($ alg $)[I]$ ) and consider the particular case $\mathcal{I}=\mathcal{P}(I)$. From (2.5) and (2.9), the equality $\mathfrak{F}[I]=\mathbb{F}^{*}(\mathcal{P}(I))$ follows. As a corollary, by (2.6) and (2.10) $\mathfrak{F}_{\mathrm{u}}[I]=\mathbb{F}_{0}^{*}(\mathcal{P}(I))$. Using (2.7) and (2.12), we obtain that $\mathfrak{F}_{\mathbf{u}}[I \mid \mathcal{J}]=\mathbb{F}_{0}^{*}(\mathcal{P}(I) \mid \mathcal{J})=\mathbf{F}_{0}^{*}(\mathcal{P}(I) \mid \mathcal{J}) \forall \mathcal{J} \in \mathcal{P}^{\prime}(\mathcal{P}(I)) . \quad$ Of $\quad$ course, $(I-$ ult $)[x]=((I, \mathcal{P}(I))-$ ult $)[x]$ under $x \in I$.

Example. Let $\mathbb{R}$ be real line. In this item, we fix $a \in \mathbb{R}, b \in] a, \infty[$, and suppose that $I=[a, b]$. Let

$$
\mathfrak{I} \triangleq\{L \in \mathcal{P}(I) \mid \exists c \in I \exists d \in I:(] c, d[\subset L) \&(L \subset[c, d])\}
$$

The family (2.17) is a semialgebra of subsets of $I$. In this item, we suppose that $\mathcal{I} \in(\mathrm{alg})[I]$ is algebra of subsets of $I=[a, b]$ generated by semialgebra $\mathfrak{I}: \mathfrak{I} \subset \mathcal{I}$ and $\forall \mathcal{L} \in(\mathrm{alg})[I]$

$$
(\mathfrak{I} \subset \mathcal{L}) \Rightarrow(\mathcal{I} \subset \mathcal{L}) .
$$

So, here $(I, \mathcal{I})$ is a measurable space with algebra of sets. Then, by statements of [9]

$$
\begin{aligned}
& \mathfrak{U}_{t}^{(-)} \triangleq\left\{J \in \mathcal{I} \mid \exists c \in\left[a, t\left[:\left[c, t[\subset J\} \in \mathbb{F}_{0, \mathbf{f}}^{*}(\mathcal{I}) \forall t \in\right] a, b\right]\right.\right. \\
& \left.\left.\left.\left.\mathfrak{U}_{t}^{(+)} \triangleq\{J \in \mathcal{I} \mid \exists c \in] t, b\right]:\right] t, c\right] \subset J\right\} \in \mathbb{F}_{0, \mathbf{f}}^{*}(\mathcal{I}) \forall t \in[a, b[
\end{aligned}
$$

We note that (see (2.15) and constructions of Section 6 in [9]) in our case

$$
\left.\left.\mathbb{F}_{0, \mathbf{f}}^{*}(\mathcal{I})=\left\{\mathfrak{U}_{t}^{(-)}: t \in\right] a, b\right]\right\} \cup\left\{\mathfrak{U}_{t}^{(+)}: t \in[a, b[\}\right.
$$

since (in our case) $\mathcal{I} \in \tilde{\pi}^{0}[I]$, by (2.16) we obtain that 
$((I, \mathcal{I})-$ ult $)[x] \in \mathbb{F}_{0}^{*}(\mathcal{I}) \forall x \in I$. Therefore, by (2.15) and (2.20) in our case

$$
\left.\left.\mathbb{F}_{0}^{*}(\mathcal{I})=\left\{\mathfrak{U}_{t}^{(-)}: t \in\right] a, b\right]\right\} \cup\left\{\mathfrak{U}_{t}^{(+)}: t \in\left[a, b[\} \cup \mathbb{F}_{0, \mathbf{t}}^{*}(\mathcal{I})\right.\right.
$$

In (2.21), we obtain exhausting representation of $\mathbb{F}_{0}^{*}(\mathcal{I})$ for considered variant of $(I, \mathcal{I})$.

\section{Elements of Topology}

For every topology $\tau \in($ top $)[\mathbf{I}]$, where $\mathbf{I}$ is a nonempty set, we obtain TS $(\mathbf{I}, \tau)$; by $(\tau$-comp $)[\mathbf{I}]$ the family of all subsets of $\mathbf{I}$ compact in TS $(\mathbf{I}, \tau)$ is denoted. Of course, $\tau \in \pi[\mathbf{I}]$. And what is more, $\tau \in(\mathrm{LAT})_{0}[\mathbf{I}]$. In addition, for every point $x \in \mathbf{I}$

$$
N_{\tau}^{0}(x) \triangleq((\mathbf{I}, \tau)-\text { ult })[x]=\{G \in \tau \mid x \in G\} \in \mathbb{F}^{*}(\tau)
$$

and, in particular, $N_{\tau}^{0}(x) \in \beta_{0}[\mathbf{I}]$; therefore,

$$
N_{\tau}(x) \triangleq(\mathbf{I}-\mathbf{f i})\left[N_{\tau}^{0}(x)\right] \in \mathfrak{F}[\mathbf{I}]
$$

is the filter of all neighborhoods (see definition of ch.I in monograph [6]) of $x$ in TS $(\mathbf{I}, \tau)$. If $A \in \mathcal{P}(E)$, then

$$
\begin{aligned}
\operatorname{cl}(A, \tau) & \triangleq\left\{x \in \mathbf{I} \mid A \cap G \neq \varnothing \forall G \in N_{\tau}^{0}(x)\right\} \\
& =\left\{x \in \mathbf{I} \mid A \cap S \neq \varnothing \forall S \in N_{\tau}(x)\right\} \in \mathbf{C}_{\mathbf{I}}[\tau]
\end{aligned}
$$

(the closure of $A$ is introduced); moreover, we sequentially introduce interior and frontier of $A$ :

$$
(\tau-\operatorname{Int})[A] \triangleq\left\{x \in \mathbf{I} \mid A \in N_{\tau}(x)\right\},(\tau-\operatorname{Fr})[A] \triangleq \operatorname{cl}(A, \tau) \backslash(\tau-\operatorname{Int})[A]
$$

Finally, $(\tau-$ dens $)[\mathbf{I}] \triangleq\{H \in \mathcal{P}(\mathbf{I}) \mid \operatorname{cl}(H, \tau)=\mathbf{I}\}$ and $(\tau-$ isol $)[\mathbf{I}] \triangleq\{x \in \mathbf{I} \mid\{x\} \in \tau\}$ are the family of all subsets of $\mathbf{I}$ every dense (in $(\mathbf{I}, \tau))$ and the set of all isolated points of $\mathbf{I}$ respectively. In correspondence with definition of ch.I of [6], we suppose that $\forall \mathcal{B} \in \beta_{0}[\mathbf{I}] \forall x \in \mathbf{I}$

$$
(\mathcal{B} \stackrel{\tau}{\Rightarrow} x) \stackrel{\operatorname{def}}{\Leftrightarrow}\left(N_{\tau}(x) \subset(\mathbf{I}-\mathbf{f i})[\mathcal{B}]\right)
$$

By (3.1) the convergence of filter bases is defined (we note that $\mathfrak{F}[\mathbf{I}] \subset \beta_{0}[\mathbf{I}]$; moreover, $\mathbb{F}^{*}(\mathcal{L}) \subset \beta_{0}[\mathbf{I}]$ under $\left.\mathcal{L} \in \pi[\mathbf{I}]\right)$. We recall that, under $J \in \mathcal{P}^{\prime}(\mathbf{I})$, topology $\left.\tau\right|_{J}=\{J \cap G: G \in \tau\} \in($ top $)[J]$ converts $J$ in subspace $\left(J,\left.\tau\right|_{J}\right)$ of TS $(\mathbf{I}, \tau)$.

We introduce the families (can - op $)[\tau] \triangleq\left\{(\tau-\right.$ Int $\left.)[F]: F \in \mathbf{C}_{\mathbf{I}}[\tau]\right\}$ and $(\operatorname{can}-\operatorname{clos})[\tau] \triangleq\{\operatorname{cl}(G, \tau): G \in \tau\}$ of all canonically open and closed sets res-pectively.

If $\left(X, \tau_{1}\right), X \neq \varnothing$, and $\left(Y, \tau_{2}\right), Y \neq \varnothing$, are TS, then

$$
C\left(X, \tau_{1}, Y, \tau_{2}\right) \triangleq\left\{f \in Y^{X} \mid f^{-1}(G) \in \tau_{1} \forall G \in \tau_{2}\right\}
$$

is the set of all continuous mappings from $\left(X, \tau_{1}\right)$ into $\left(Y, \tau_{2}\right)$; recall that, for $g \in C\left(X, \tau_{1}, Y, \tau_{2}\right)$ and $K \in\left(\tau_{1}-\mathrm{comp}\right)[X]$, the property 
$g^{1}(K) \in\left(\tau_{2}-\right.$ comp $)[Y]$ is realized. We note that for every $f \in Y^{X}$ and a family $\mathcal{X} \in \mathcal{P}^{\prime}(\mathcal{P}(X))$, the (nonempty) family

$$
f^{1}[\mathcal{X}] \triangleq\left\{f^{1}(A): A \in \mathcal{X}\right\} \in \mathcal{P}^{\prime}(\mathcal{P}(Y))
$$

is defined. Moreover, for $g \in Y^{X}$ and $\mathcal{B} \in \beta_{0}[X]$, we obtain that $g^{1}[\mathcal{B}] \in \beta_{0}[Y]$.

Now, we consider some topological properties of ultrafilter space for a fixed $\pi$-system. So, we fix until end of this section a nonempty set $I$ and a $\pi$-system $\mathcal{I} \in \pi[I]$. Using (2.11), in the form of

$$
(\mathbb{U F})[I ; \mathcal{I}] \triangleq\left\{\Phi_{\mathcal{I}}(L): L \in \mathcal{I}\right\},
$$

we obtain the base of a topology of $\mathbb{F}_{0}^{*}(\mathcal{I})$. This topology is defined by the rule

$$
\begin{aligned}
\mathbf{T}_{\mathcal{I}}^{*}[I] & \triangleq\{\bigcup\}((\mathbb{U F})[I ; \mathcal{I}]) \\
& =\left\{\mathbb{G} \in \mathcal{P}\left(\mathbb{F}_{0}^{*}(\mathcal{I})\right) \mid \forall \mathcal{U} \in \mathbb{G} \exists U \in \mathcal{U}: \Phi_{\mathcal{I}}(U) \subset \mathbb{G}\right\}
\end{aligned}
$$

In addition, under $J \in \mathcal{I}$, the property

$$
\Phi_{\mathcal{I}}(J) \in \mathbf{T}_{\mathcal{I}}^{*}[I] \cap \mathbf{C}_{\mathbb{F}_{0}^{*}(\mathcal{I})}\left[\mathbf{T}_{\mathcal{I}}^{*}[I]\right]
$$

is realized (this is the simple corollary of (2.11)). In the form of

$$
\left(\mathbb{F}_{0}^{*}(\mathcal{I}), \mathbf{T}_{\mathcal{I}}^{*}[I]\right)
$$

a zero-dimensional Hausdorff TS is realized.

Remark 3.1. If $\mathcal{I} \in(\mathrm{alg})[I]$, then (3.3) is a compactum (compact Hausdorff TS). In this case, (3.3) is the Stone space. For this case,

$$
(\mathbb{U F})[I ; \mathcal{I}]=\mathbf{T}_{\mathcal{I}}^{*}[I] \cap \mathbf{C}_{\mathbb{F}_{0}^{*}(\mathcal{I})}\left[\mathbf{T}_{\mathcal{I}}^{*}[I]\right]
$$

So, we obtain (see (3.4)) the important particular case of (3.2), (3.3).

Until end of this section, we suppose that $\mathcal{I} \in \tilde{\pi}^{0}[I]$. By (2.16) we obtain the mapping

$$
x \mapsto((I, \mathcal{I})-\text { ult })[x]: I \rightarrow \mathbb{F}_{0}^{*}(\mathcal{I})
$$

denoted as $((I, \mathcal{I})-$ ult $)[\cdot]$. In addition, $\mathbb{F}_{0, \mathrm{t}}^{*}(\mathcal{I}) \subset \mathbb{F}_{0}^{*}(\mathcal{I})$. Now, we recall some statements of [10] connected with (3.5). Of course, under $S \in \mathcal{P}(I)$, in the form of

$$
((I, \mathcal{I})-\text { ult })[\cdot]^{1}(S)=\{((I, \mathcal{I})-\text { ult })[x]: x \in S\} \in \mathcal{P}\left(\mathbb{F}_{0, \mathrm{t}}^{*}(\mathcal{I})\right)
$$

we obtain the image of $S$ under operation (3.5). Then (see [10])

$$
\mathbf{F}_{0}^{*}[\mathcal{I} \mid A]=\operatorname{cl}\left(((I, \mathcal{I})-\text { ult })[\cdot]^{1}(A), \mathbf{T}_{\mathcal{I}}^{*}[I]\right) \forall A \in \mathcal{P}(I)
$$

As a corollary, $\Phi_{\mathcal{I}}(L)=\operatorname{cl}\left(((I, \mathcal{I})-\right.$ ult $\left.)[\cdot]^{1}(L), \mathbf{T}_{\mathcal{I}}^{*}[I]\right) \forall L \in \mathcal{I}$. Finally,

$$
\mathbb{F}_{0}^{*}(\mathcal{I})=\Phi_{\mathcal{I}}(I)=\operatorname{cl}\left(\mathbb{F}_{0, \mathrm{t}}^{*}(\mathcal{I}), \mathbf{T}_{\mathcal{I}}^{*}[I]\right)
$$

So, $\mathbb{F}_{0, \mathbf{t}}^{*}(\mathcal{I}) \in\left(\mathbf{T}_{\mathcal{I}}^{*}[I]-\right.$ dens $)\left[\mathbb{F}_{0}^{*}(\mathcal{I})\right]$. We recall that $(\mathrm{alg})[I] \subset \tilde{\pi}^{0}[I]$ (see (2.3)). Therefore, (3.6) and (3.7) are fulfilled under $\mathcal{I} \in($ alg $)[I]$. 


\section{Attainability under Constraints of Asymptotic Character}

Returning to Section 1, we preserve the designation $E$ for the space of usual solutions. Families $\mathcal{E} \in \mathcal{P}^{\prime}(\mathcal{P}(E))$ are considered as constraints of asymptotic character (see Section 1). Moreover, in this section, we fix a TS $(Y, \tau), Y \neq \varnothing$ and a mapping $f \in Y^{E}$. We discuss construction of AS in $(Y, \tau)$. These AS can be used as basic or as auxiliary. In Section 1, nets in $E$ were used as distinctive "asymptotic solurions". But, now the analogous employment of filters and ultrafilters is more appropriate. The equivalence of the representations with employment of nets and ultrafilters was noted (in particular) in $\$ 3$ of [7]. Now, we discuss the representation realized in class of ultrafilters. So, by definition of $\$ 3$ of $[7]$

$$
\begin{aligned}
(\text { as })[E ; Y ; \tau ; f ; \mathcal{E}] & \triangleq\left\{y \in Y \mid \exists \mathcal{U} \in \mathfrak{F}_{\mathbf{u}}^{0}[E \mid \mathcal{E}]: f^{1}[\mathcal{U}] \stackrel{\tau}{\Rightarrow} y\right\} \\
& =\bigcap_{\Sigma \in\left\{\bigcap_{\}_{\mathbf{f}}}(\mathcal{E})\right.} \operatorname{cl}\left(f^{1}(\Sigma), \tau\right) \in \mathbf{C}_{Y}[\tau] \forall \mathcal{E} \in \mathcal{P}^{\prime}(\mathcal{P}(E))
\end{aligned}
$$

We note that the following particular case is useful:

$$
\text { (as) }[E ; Y ; \tau ; f ; \mathcal{E}]=\bigcap_{\Sigma \in \mathcal{E}} \operatorname{cl}\left(f^{1}(\Sigma), \tau\right) \forall \mathcal{E} \in \beta[E]
$$

In connection with (4.2), we recall discussion in Section 1 (see (1.2)). We consider the sets (4.1) and (4.2) as AS corresponding to given nonempty family of subsets of $E$ (we recall that, under employment of nets in $E$, we obtain the equivalent representation of AS; in this connection, see constructions of $\$ 3$ in [7]). By (4.1) the following singularity is realized: ultrafilters play the role of approximate solutions of J.Warga.

\section{Representations of Attraction Sets}

In this section, we consider some transformations of AS. In addition, we fix a TS $(\mathbf{H}, \mathbf{t}), \mathbf{H} \neq \varnothing$ and $\mathbf{h} \in \mathbf{H}^{E}$ (see Section 1). Under $\mathcal{E} \in \mathcal{P}^{\prime}(\mathcal{P}(E))$, we consider

$$
\text { (as) }[E ; \mathbf{H} ; \mathbf{t} ; \mathbf{h} ; \mathcal{E}] \in \mathbf{C}_{\mathbf{H}}[\mathbf{t}]
$$

as the basic AS. Namely, in the case when $\mathcal{E}$ defines constraints of asymptotic character, (5.1) is our goal set. For investigation of the set (5.1) we can exploit auxiliary AS. We note the following general property: if $(Y, \tau), Y \neq \varnothing$ is a TS, $m \in Y^{E}$ and $g \in C(Y, \tau, \mathbf{H}, \mathbf{t})$, then

$(\mathbf{h}=g \circ m) \Rightarrow\left(g^{1}((\mathbf{a s})[E ; Y ; \tau ; m ; \mathcal{E}]) \subset(\mathbf{a s})[E ; \mathbf{H} ; \mathbf{t} ; \mathbf{h} ; \mathcal{E}] \forall \mathcal{E} \in \mathcal{P}^{\prime}(\mathcal{P}(E))\right)$.

This property is established very simply with employment of continuity of the mapping $g$ and (4.1). From relations (2.8.1), (2.8.2), and Proposition 3.3.1 of [11], under conditions with respect to $(Y, \tau, m, g)$ used in (2), we obtain that

$$
\begin{aligned}
& \left(\left(g^{1}(F) \in \mathbf{C}_{\mathbf{H}}[\mathbf{t}] \forall F \in \mathbf{C}_{Y}[\tau]\right)\right. \\
& \left.\&\left(g^{-1}(\{h\}) \in(\tau-\text { comp })[Y] \forall h \in \mathbf{H}\right) \&(\mathbf{h}=g \circ m)\right) \\
& \Rightarrow\left(g^{1}((\mathbf{a s})[E ; Y ; \tau ; m ; \mathcal{E}])=(\mathbf{a s})[E ; \mathbf{H} ; \mathbf{t} ; \mathbf{h} ; \mathcal{E}] \forall \mathcal{E} \in \mathcal{P}^{\prime}(\mathcal{P}(E))\right) ;
\end{aligned}
$$


of course, we use (4.1). We recall that the continuous mapping $g$ satisfying to two first conditions in premise of the implication (5.3) is called almost perfect; see Section 3.7 of monograph [12]. In connection with (5.3), we note the important particular case of such mapping (see formula (2.8.7) of [11]): if $(Y, \tau), Y \neq \varnothing$ is a compact TS and $(\mathbf{H}, \mathbf{t})$ is a Hausdorff space, then $\forall g \in C(Y, \tau, \mathbf{H}, \mathbf{t})$

$$
\left(g^{1}(F) \in \mathbf{C}_{\mathbf{H}}[\mathbf{t}] \forall F \in \mathbf{C}_{Y}[\tau]\right) \&\left(g^{-1}(\{h\}) \in(\tau-\text { comp })[Y] \forall h \in \mathbf{H}\right)
$$

Therefore, the natural compactified case arises. In this connection, in the folowing, we suppose always that

$$
(\mathbf{H}, \mathbf{t})
$$

is a Hausdorff TS. The case of such spaces (5.5) is sufficient for majority applications. Then (in our case of TS (5.5)) for every compact TS $(Y, \tau), Y \neq \varnothing, m \in Y^{E}$ and $g \in C(Y, \tau, \mathbf{H}, \mathbf{t})$, the implication

$$
(\mathbf{h}=g \circ m) \Rightarrow\left((\mathbf{a s})[E ; \mathbf{H} ; \mathbf{t} ; \mathbf{h} ; \mathcal{E}]=g^{1}((\mathbf{a s})[E ; Y ; \tau ; m ; \mathcal{E}]) \forall \mathcal{E} \in \mathcal{P}^{\prime}(\mathcal{P}(E))\right)
$$

In connection with the given important property (5.6), we introduce the special notion of a compactifier. Namely [4], we call compactifier every four $(Y, \tau, m, g)$ such that $(Y, \tau)$ is a compact TS, $Y \neq \varnothing, m \in Y^{E}, g \in C(Y, \tau, \mathbf{H}, \mathbf{t})$ and $\mathbf{h}=g \circ m$. From (5.6), we obtain that for every compactifier $(Y, \tau, m, g)$

$$
(\mathbf{a s})[E ; \mathbf{H} ; \mathbf{t} ; \mathbf{h} ; \mathcal{E}]=g^{1}((\mathbf{a s})[E ; Y ; \tau ; m ; \mathcal{E}]) \forall \mathcal{E} \in \mathcal{P}^{\prime}(\mathcal{P}(E)) \text {. }
$$

So, every compactifier realizes the representation of the basic AS as continuous image of auxiliary AS in compact space. In connection with (5.7), we note that (closed) set

$$
\tilde{\mathbf{H}} \triangleq \operatorname{cl}\left(\mathbf{h}^{1}(E), \mathbf{t}\right) \in \mathbf{C}_{\mathbf{H}}[\mathbf{t}]
$$

has the following obvious property (see (4.1)):

$$
\text { (as) }[E ; \mathbf{H} ; \mathbf{t} ; \mathbf{h} ; \mathcal{E}] \subset \tilde{\mathbf{H}} \forall \mathcal{E} \in \mathcal{P}^{\prime}(\mathcal{P}(E)) \text {; }
$$

moreover, under $\Sigma \in \mathcal{P}(E)$, from (5.8), we obtain that

$$
\operatorname{cl}\left(\mathbf{h}^{1}(\Sigma), \mathbf{t}\right)=\operatorname{cl}\left(\mathbf{h}^{1}(\Sigma), \tilde{\mathbf{t}}\right)
$$

where (here and later) the designation

$$
\left.\tilde{\mathbf{t}} \triangleq \mathbf{t}\right|_{\tilde{\mathbf{H}}} \in(\text { top })[\tilde{\mathbf{H}}]
$$

is used. So, by (4.1) and (5.9) we obtain that, for Hausdorff TS

$$
(\tilde{\mathbf{H}}, \tilde{\mathbf{t}})
$$

(subspace of the initial TS (5.5)), the following equalities

$$
(\mathbf{a s})[E ; \mathbf{H} ; \mathbf{t} ; \mathbf{h} ; \mathcal{E}]=(\mathbf{a s})[E ; \tilde{\mathbf{H}} ; \tilde{\mathbf{t}} ; \mathbf{h} ; \mathcal{E}] \forall \mathcal{E} \in \mathcal{P}^{\prime}(\mathcal{P}(E))
$$

are realized; of course, we use the next obvious property: $\mathbf{h} \in \tilde{\mathbf{H}}^{E} \quad$ (see (5.8)). In connection with (5.12), we suppose that (by (5.10)) the replacement (5.5) $\rightarrow$ 
(5.11) is realized.

Now, we consider the corresponding reduction of compactifiers defined in terms of TS (5.5).

Proposition 5.1. If $(Y, \tau, m, g)$ is a compactifier, then $\tilde{\mathbf{H}} \subset g^{1}(Y)$.

Proof. Indeed, we obtain that

$$
\begin{aligned}
\tilde{\mathbf{H}} & =\operatorname{cl}\left(\mathbf{h}^{1}(E), \mathbf{t}\right)=\operatorname{cl}\left((g \circ m)^{1}(E), \mathbf{t}\right) \\
& =\operatorname{cl}\left(g^{1}\left(m^{1}(E)\right), \mathbf{t}\right) \subset \operatorname{cl}\left(g^{1}(Y), \mathbf{t}\right)=g^{1}(Y)
\end{aligned}
$$

where (5.4) is used (really, $g^{1}(Y)$ is a closed set).

Proposition 5.2. If $(Y, \tau, m, g)$ is a compactifier, then

$$
\left(\operatorname{cl}\left(m^{1}(E), \tau\right),\left.\tau\right|_{\mathrm{cl}\left(m^{1}(E), \tau\right)}, m,\left(g \mid \operatorname{cl}\left(m^{1}(E), \tau\right)\right)\right)
$$

is compactifier also.

The corresponding proof is given in Section 4 of [4].

In the following, we call a compactifier $(Y, \tau, m, g)$ dense in the case when $\operatorname{cl}\left(m^{1}(E), \tau\right)=Y$. By Proposition 5.2 we obtain that (as in Proposition 4.1 of [4]) every compactifier assumes transformation to a dense compactifier. Therefore, later, we consider only dense compactifiers. It is obvious the following

Proposition 5.3. If $(Y, \tau, m, g)$ is a dense compactifier, then $g$ is a surjection from $Y$ onto $\tilde{\mathbf{H}}$.

Proof. Indeed, by (5.4) we obtain that

$$
\begin{aligned}
g^{1}(Y) & =g^{1}\left(\operatorname{cl}\left(m^{1}(E), \tau\right)\right)=\operatorname{cl}\left(g^{1}\left(m^{1}(E)\right), \mathbf{t}\right) \\
& =\operatorname{cl}\left((g \circ m)^{1}(E), \mathbf{t}\right)=\operatorname{cl}\left(\mathbf{h}^{1}(E), \mathbf{t}\right)=\tilde{\mathbf{H}}
\end{aligned}
$$

(in (5.13) we use constructions of [11]; in particular, see relations (2.8.1)(2.8.4) and (2.8.7) of [11]).

Corollary 5.1. If $(Y, \tau, m, g)$ is a dense compactifier for which $g$ is an injective mapping from $Y$ in $\mathbf{H}$, then $g$ is a homeomorphism from TS $(Y, \tau)$ onto TS (5.11).

Proof. Let all conditions with respect to $(Y, \tau, m, g)$ are fulfilled. Then, by Proposition 5.3 the mapping $g$ is a bijection from $Y$ onto $\tilde{\mathbf{H}}$. Moreover, let $\tilde{\mathbb{G}} \in \tilde{\mathbf{t}}$. Using (5.10), we choose $\mathbb{G} \in \mathbf{t}$ for which $\tilde{\mathbb{G}}=\tilde{\mathbf{H}} \cap \mathbb{G}$. Then

$$
\begin{aligned}
g^{-1}(\tilde{\mathbb{G}}) & =\{y \in Y \mid g(y) \in \tilde{\mathbb{G}}\}=\{y \in Y \mid g(y) \in \tilde{\mathbf{H}} \cap \tilde{\mathbb{G}}\} \\
& =\{y \in Y \mid g(y) \in \mathbb{G}\}=g^{-1}(\mathbb{G}) \in \tau
\end{aligned}
$$

(we recall the obvious property: for $y \in Y$ the inclusion $g(y) \in \tilde{\mathbf{H}}$ is realized; see Proposition 5.3). Since the choice of $\tilde{\mathbb{G}}$ was arbitrary, from (5.14), the property $g \in C(Y, \tau, \tilde{\mathbf{H}}, \tilde{\mathbf{t}})$ follows. But, $(Y, \tau)$ is compact and (5.11) is Hausdorff TS. Therefore, $g$ is a homeomorphism from $(Y, \tau)$ onto (5.11) (indeed, $g$ is a continuous bijection from compact TS onto Hausdorff TS; now, we use Theorem 3.1.13 of monograph [12]). 


\section{Compactifiers and Quotient Topologies}

We recall the known notion: if $(X, \tau), X \neq \varnothing$ is a TS, $Y$ is a nonempty set, and $f \in Y^{X}$, then

$$
\left\{G \in \mathcal{P}(Y) \mid f^{-1}(G) \in \tau\right\} \in(\text { top })[Y]
$$

is called quotient topology on $Y$ (this topology was introduced by P. S. Alexandroff and H. Hopf). In supplement to (6.1), we note the obvious property: for every nonempty sets $X$ and $Y$, a surjection $g$ from $X$ onto $Y$, and a set $B \in \mathcal{P}(Y)$, the equality

$$
g^{1}\left(g^{-1}(B)\right)=B
$$

is fulfilled ((6.2) is known property of surjections).

Remark 6.1. Let $(X, \tau), X \neq \varnothing$ be TS, $Y$ be a nonempty set, and $f \in Y^{X}$. Moreover, we suppose that $\theta$ is topology (6.1). Then,

$$
\mathbf{C}_{Y}[\theta] \triangleq\left\{F \in \mathcal{P}(Y) \mid f^{-1}(F) \in \mathbf{C}_{X}[\tau]\right\}
$$

For more brief presentation, we introduce some new designations. So, for every nonempty sets $X$ and $Y$, by $Y_{(*)}^{X}$ we denote the set of all surjections from $X$ onto $Y$. If $\left(X, \tau_{1}\right), X \neq \varnothing$ and $\left(Y, \tau_{2}\right), Y \neq \varnothing$ are two TS, then

$$
\begin{gathered}
C_{\mathrm{cl}}\left(X, \tau_{1}, Y, \tau_{2}\right) \triangleq\left\{f \in C\left(X, \tau_{1}, Y, \tau_{2}\right) \mid f^{1}(F) \in \mathbf{C}_{Y}\left[\tau_{2}\right] \forall F \in \mathbf{C}_{X}\left[\tau_{1}\right]\right\} \\
C_{\text {op }}\left(X, \tau_{1}, Y, \tau_{2}\right) \triangleq\left\{f \in C\left(X, \tau_{1}, Y, \tau_{2}\right) \mid f^{1}(G) \in \tau_{2} \forall G \in \tau_{1}\right\}
\end{gathered}
$$

In (6.3) and (6.4), closed and open continuous mappings are introduced. In connection with (6.3) and (6.4), we note that (see ch.3 of monograph [13]) for every TS $\left(X, \tau_{1}\right), X \neq \varnothing$ and $\left(Y, \tau_{2}\right), Y \neq \varnothing$, the following property takes place: if $f \in Y_{(*)}^{X}$, then

$$
\begin{aligned}
& \left(\left(f \in C_{\mathrm{cl}}\left(X, \tau_{1}, Y, \tau_{2}\right)\right) \vee\left(f \in C_{\mathrm{op}}\left(X, \tau_{1}, Y, \tau_{2}\right)\right)\right) \\
& \Rightarrow\left(\tau_{2}=\left\{G \in \mathcal{P}(Y) \mid f^{-1}(G) \in \tau_{1}\right\}\right)
\end{aligned}
$$

As a simple corollary, the following property is realized: if $\left(X, \tau_{1}\right), X \neq \varnothing$ is a compact TS, $\left(Y, \tau_{2}\right), Y \neq \varnothing$ is a Hausdorff TS, and $f \in C\left(X, \tau_{1}, Y, \tau_{2}\right) \cap Y_{(*)}^{X}$, then

$$
\tau_{2}=\left\{G \in \mathcal{P}(Y) \mid f^{-1}(G) \in \tau_{1}\right\}
$$

We note that conditions realizing (6.5) correspond to variant used under employment of dense compactifiers (see Proposition 5.3).

Proposition 6.1. If $(Y, \tau, m, g)$ is a dense compactifier, then

$$
\tilde{\mathbf{t}}=\left\{G \in \mathcal{P}(\tilde{\mathbf{H}}) \mid g^{-1}(G) \in \tau\right\}
$$

Proof. From Proposition 5.3 and constructions similar to (5.14), we obtain that 


$$
g \in C(Y, \tau, \tilde{\mathbf{H}}, \tilde{\mathbf{t}}) \cap \tilde{\mathbf{H}}_{(*)}^{Y}
$$

where $(Y, \tau)$ is a compact and $(\tilde{\mathbf{H}}, \tilde{\mathbf{t}})$ is a Hausdorff TS. So, (6) follows from (6.5).

We obtain that the closed subspace of $(\mathbf{H}, \tau)$ corresponding to (5.8) is TS with quotient topology with respect to every dense compactifier. We note two obvious (but useful) general statements.

Remark 6.2. Let $\left(X, \tau_{1}\right), X \neq \varnothing$ and $\left(Y, \tau_{2}\right), Y \neq \varnothing$ are TS. Moreover, let

$$
f \in C_{\mathrm{cl}}\left(X, \tau_{1}, Y, \tau_{2}\right) \cap Y_{(*)}^{X}
$$

Then, the equality $f^{1}\left[\mathbf{C}_{X}\left[\tau_{1}\right]\right]=\mathbf{C}_{Y}\left[\tau_{2}\right]$ is realized. We consider the brief scheme of the proof.

The inclusion $f^{1}\left[\mathbf{C}_{X}\left[\tau_{1}\right]\right] \subset \mathbf{C}_{Y}\left[\tau_{2}\right]$ follows from (6.3) and (6.7). Let $\Phi \in \mathbf{C}_{Y}\left[\tau_{2}\right]$. By (6.2) and (6.7) $\Phi=f^{1}\left(f^{-1}(\Phi)\right)$, where $f^{-1}(\Phi) \in \mathbf{C}_{X}\left[\tau_{1}\right]$. So, $\Phi \in f^{1}\left[\mathbf{C}_{X}\left[\tau_{1}\right]\right]$. Since the choice of $\Phi$ was arbitrary, we obtain the inclusion $\mathbf{C}_{Y}\left[\tau_{2}\right] \subset f^{1}\left[\mathbf{C}_{X}\left[\tau_{1}\right]\right]$.

Remark 6.3. Let $\left(X, \tau_{1}\right)$ and $\left(Y, \tau_{2}\right)$ correspond to Remark 6.2. Moreover, let

$$
f \in C_{\text {op }}\left(X, \tau_{1}, Y, \tau_{2}\right) \cap Y_{(*)}^{X}
$$

Then, $f^{1}\left[\tau_{1}\right]=\tau_{2}$. Indeed, by (6.4) and (6.8) $f^{1}\left[\tau_{1}\right] \subset \tau_{2}$. Let $\mathbb{G} \in \tau_{2}$. Then, by (6.2) and (6.8)

$$
\mathbb{G}=f^{1}\left(f^{-1}(\mathbb{G})\right)
$$

where $f^{-1}(\mathbb{G}) \in \tau_{1}$. Therefore, $\mathbb{G} \in f^{1}\left[\tau_{1}\right]$. The inclusion $\tau_{2} \subset f^{1}\left[\tau_{1}\right]$ is established.

Proposition 6.2. If $(Y, \tau, m, g)$ is a dense compactifier, then

$$
g^{1}\left[\mathbf{C}_{Y}[\tau]\right]=\mathbf{C}_{\tilde{\mathbf{H}}}[\tilde{\mathbf{t}}]
$$

Proof. By Proposition $5.3 g \in \tilde{\mathbf{H}}_{(*)}^{Y}$. In addition, similarly to (5.14), we obtain that $g \in C(Y, \tau, \tilde{\mathbf{H}}, \tilde{\mathbf{t}})$. Since $(Y, \tau)$ is compact and $(\tilde{\mathbf{H}}, \tilde{\mathbf{t}})$ is a Hausdorff TS, $g \in C_{\mathrm{cl}}(Y, \tau, \tilde{\mathbf{H}}, \tilde{\mathbf{t}})$; see (2.8.2) and (2.8.7) in monograph [11]. From Remark 6.2, the equality (6.9) follows.

Proposiotion 6.3. If $(Y, \tau, m, g)$ is a dense compactifier, then

$$
g^{1}[(\tau-\text { comp })[Y]]=(\tilde{\mathbf{t}}-\text { comp })[\tilde{\mathbf{H}}]
$$

Proof. We recall that $g \in C(Y, \tau, \tilde{\mathbf{H}}, \tilde{\mathbf{t}})$ (see discussion in connection with (5.14)). Then, $g^{1}(K) \in(\tilde{\mathbf{t}}-$ comp $)[\tilde{\mathbf{H}}] \forall K \in(\tau$-comp $)[Y]$. Therefore,

$$
g^{1}[(\tau-\text { comp })[Y]] \subset(\tilde{\mathbf{t}}-\operatorname{comp})[\tilde{\mathbf{H}}]
$$

Let $\tilde{\mathbf{K}} \in(\tilde{\mathbf{t}}-\operatorname{comp})[\tilde{\mathbf{H}}]$. Then, $\tilde{\mathbf{K}} \in \mathbf{C}_{\tilde{\mathbf{H}}}[\tilde{\mathbf{t}}]$ (indeed, (5.11) is a Hausdorff TS). By Proposition 6.2 $\tilde{\mathbf{K}} \in g^{1}\left[\mathbf{C}_{Y}[\tau]\right]$, where $\mathbf{C}_{Y}[\tau] \subset(\tau$-comp $)[Y]$ since $(Y, \tau)$ is a compact TS. Then, $\tilde{\mathbf{K}} \in g^{1}[(\tau-$ comp $)[Y]]$. So, the inclusion

$$
(\tilde{\mathbf{t}}-\text { comp })[\tilde{\mathbf{H}}] \subset g^{1}[(\tau-\text { comp })[Y]]
$$


is realized. Using (6.11), we obtain (6.10).

Remark 6.4. We note some properties connected with Proposition 6.1. Let $(Y, \tau, m, g)$ be a fixed dense compactifier. Then

$$
\tilde{\mathbf{t}} \subset g^{1}[\tau]
$$

Indeed, let $\tilde{G} \in \tilde{\mathbf{t}}$. Since $g \in \tilde{\mathbf{H}}_{(*)}^{Y}$ by Proposition 5.3, we obtain that $\tilde{G}=g^{1}\left(g^{-1}(\tilde{G})\right)$, where $g^{-1}(\tilde{G}) \in \tau$ by continuity of $g$ (see (5.14)). As a result $\tilde{G} \in g^{1}[\tau]$. Since the choice of $\tilde{G}$ was arbitrary, the required inclusion (6.12) is realized. The analogous construction realizes the inclusion

$$
\tilde{\mathbf{t}} \cap \mathbf{C}_{\tilde{\mathbf{H}}}[\tilde{\mathbf{t}}] \subset g^{1}\left[\tau \cap \mathbf{C}_{Y}[\tau]\right]
$$

where (6.2) and continuity of $g$ are used. Indeed, for $\Gamma \in \tilde{\mathbf{t}} \cap \mathbf{C}_{\tilde{\mathbf{H}}}[\tilde{\mathbf{t}}]$, we obtain that $g^{-1}(\Gamma) \in \tau \cap \mathbf{C}_{Y}[\tau]$ (continuity of $g$ is used) and $\Gamma \subset g^{1}\left(g^{-1}(\Gamma)\right.$ ) by (6.2). So, $\Gamma \in g^{1}\left[\tau \cap \mathbf{C}_{Y}[\tau]\right]$. The inclusion (6.13) is established.

\section{Some Transformations of Compactifiers}

In this section, we fix a dense compactifier $(Y, \tau, m, g)$. Then

$$
g \in C(Y, \tau, \tilde{\mathbf{H}}, \tilde{\mathbf{t}}) \cap \tilde{\mathbf{H}}_{(*)}^{Y}
$$

(we use reasons similar to (5.14)). Now, we consider the natural transformation of $(Y, \tau, m, g)$ in injective compactifier (see Proposition 5.3). In addition, constructions of [4] are used. The family

$$
\mathcal{Y} \triangleq\left\{g^{-1}(\{z\}): z \in \tilde{\mathbf{H}}\right\}=\left\{g^{-1}(\{g(y)\}): y \in Y\right\} \in \mathcal{P}^{\prime}\left(\mathcal{P}^{\prime}(Y)\right)
$$

is a partition of $Y$; of course, $\mathcal{Y}$ (7.2) generates the following equivalence relation $\equiv$ on $Y$ by the rule: $\forall y_{1} \in Y \forall y_{2} \in Y$

$$
\left(y_{1} \equiv y_{2}\right) \stackrel{\text { def }}{\Leftrightarrow}\left(g\left(y_{1}\right)=g\left(y_{2}\right)\right)
$$

In addition, $\quad\{\tilde{y} \in Y \mid y \equiv \tilde{y}\}=g^{-1}(\{g(y)\}) \in \mathcal{P}^{\prime}(Y) \forall y \in Y$. We note that $\forall \mathbb{Y} \in \mathcal{Y} \exists ! h \in \tilde{\mathbf{H}}: g(y)=h \quad \forall y \in \mathbb{Y}$. Therefore, we introduce the mapping $\sigma \in \tilde{\mathbf{H}}^{\mathcal{Y}}$ by the next rule:

$$
\sigma(\mathbb{Y}) \triangleq g(y) \forall \mathbb{Y} \in \mathcal{Y} \forall y \in \mathbb{Y}
$$

From (7.1) and (7.4), we obtain that $\sigma \in \tilde{\mathbf{H}}_{(*)}^{\mathcal{Y}}$. And what is more, $\sigma$ is a bijection from $\mathcal{Y}$ onto $\tilde{\mathbf{H}}$.

Now, we obtain that $\mathbf{p} \triangleq\left(g^{-1}(\{g(y)\})\right)_{y \in Y} \in \mathcal{Y}_{(*)}^{Y}$. So, the canonical projection from $Y$ onto the corresponding factor-space is defined. In addition,

$$
g=\sigma \circ \mathbf{p}
$$

(the canonical expansion of $g$ ). Indeed, let $y_{*} \in Y$ and $D_{*} \triangleq g^{-1}\left(\left\{g\left(y_{*}\right)\right\}\right)$. In addition, $\mathbf{p}\left(y_{*}\right)=g^{-1}\left(\left\{g\left(y_{*}\right)\right\}\right)=D_{*} \in \mathcal{Y}$;

$$
(\sigma \circ \mathbf{p})\left(y_{*}\right)=\sigma\left(\mathbf{p}\left(y_{*}\right)\right)=\sigma\left(D_{*}\right)
$$


Since $y_{*} \in D_{*}$, by (7.4) we obtain that $\sigma\left(D_{*}\right)=g\left(y_{*}\right)$. So, by (7.6) $(\sigma \circ \mathbf{p})\left(y_{*}\right)=g\left(y_{*}\right)$. Since the choice of $y_{*}$ was arbitrary, the property

$$
(\sigma \circ \mathbf{p})(y)=g(y) \forall y \in Y
$$

is realized. As a result, we obtain (7.5).

Following constructions of Section 2.4 of monograph [12], we equip (see [4]) the set $\mathcal{Y}$ with quotient topology. Namely,

$$
\mathcal{T} \triangleq\left\{\mathbb{G} \in \mathcal{P}(\mathcal{Y}) \mid \mathbf{p}^{-1}(\mathbb{G}) \in \tau\right\} \in(\text { top })[\mathcal{Y}]
$$

So, we obtain TS $(\mathcal{Y}, \mathcal{T})$ for which

$$
\mathbf{p} \in C(Y, \tau, \mathcal{Y}, \mathcal{T})
$$

Since $\mathbf{p}^{1}(Y)=\mathcal{Y}$, the following property is realized: $(\mathcal{Y}, \mathcal{T})$ is a compact TS (we apply the continuity of $\mathbf{p}$ realized in $(7.8)$ and compactness of $(Y, \tau)$ ).

Now, we note that (by the choice of $(Y, \tau, m, g)) \quad \mathbf{p} \circ m \in \mathcal{Y}^{E}$ and

$$
\sigma \circ(\mathbf{p} \circ m)=\sigma \circ \mathbf{p} \circ m=(\sigma \circ \mathbf{p}) \circ m=g \circ m=\mathbf{h}
$$

Finally, $\sigma \in C(\mathcal{Y}, \mathcal{T}, \tilde{\mathbf{H}}, \tilde{\mathbf{t}})$. Indeed, let $\mathbb{G} \in \tilde{\mathbf{t}}$. By (7.1) we obtain that $g^{-1}(\mathbb{G}) \in \tau$. By $(7.5)$

$$
g^{-1}(\mathbb{G})=(\sigma \circ \mathbf{p})^{-1}(\mathbb{G})=\mathbf{p}^{-1}\left(\sigma^{-1}(\mathbb{G})\right)
$$

where $\sigma^{-1}(\mathbb{G}) \in \mathcal{P}(\mathcal{Y})$. Since $\mathbf{p}^{-1}\left(\sigma^{-1}(\mathbb{G})\right) \in \tau$, by (7.7) $\sigma^{-1}(\mathbb{G}) \in \mathcal{T}$. Since the choice of $\mathbb{G}$ was arbitrary also, the required continuity property is established. As a result,

$$
\sigma \in C(\mathcal{Y}, \mathcal{T}, \tilde{\mathbf{H}}, \tilde{\mathbf{t}}) \cap \tilde{\mathbf{H}}_{(*)}^{\mathcal{Y}}
$$

In particular, $(\mathcal{Y}, \mathcal{T}, \mathbf{p} \circ m, \sigma)$ is a compactifier (indeed, by (7.10) the obvious property $\sigma \in C(\mathcal{Y}, \mathcal{T}, \mathbf{H}, \mathbf{t})$ follows). And what is more, $\sigma$ is a continuous bijection from $\mathcal{Y}$ onto $\tilde{\mathbf{H}}$. Since $(\mathcal{Y}, \mathcal{T})$ is a compact space and $(\tilde{\mathbf{H}}, \tilde{\mathbf{t}})$ is a Hausdorff space, the mapping $\sigma$ is a homeomorphism from $(\mathcal{Y}, \mathcal{T})$ onto $(\tilde{\mathbf{H}}, \tilde{\mathbf{t}})$. Then, $(\mathcal{Y}, \mathcal{T}, \mathbf{p} \circ m, \sigma)$ is an injective compactifier. We note the density property of this compactifier.

Indeed, by the choice of $(Y, \tau, m, g)$ the equality $Y=\mathrm{cl}\left(m^{1}(E), \tau\right)$. Then, by (7.8)

$$
\mathcal{Y}=\mathbf{p}^{1}(Y)=\mathbf{p}^{1}\left(\operatorname{cl}\left(m^{1}(E), \tau\right)\right) \subset \operatorname{cl}\left(\mathbf{p}^{1}\left(m^{1}(E)\right), \mathcal{T}\right) \subset \mathcal{Y}
$$

So, $\operatorname{cl}\left((\mathbf{p} \circ m)^{1}(E), \mathcal{T}\right)=\mathcal{Y}$. We obtain that $(\mathcal{Y}, \mathcal{T}, \mathbf{p} \circ m, \sigma)$ is an injective dense compactifier.

In addition, $(\mathcal{Y}, \mathcal{T})$ is a Hausdorff TS. Of course, this property follows from homeomorphism of $\sigma$ and separability of $(\tilde{\mathbf{H}}, \tilde{\mathbf{t}})$. So, $(\mathcal{Y}, \mathcal{T})$ is compactum. Moreover, $\tilde{\mathbf{H}}=\sigma^{1}(\mathcal{Y})$ and, as a corollary, TS $(\tilde{\mathbf{H}}, \tilde{\mathbf{t}})$ is a compactum also.

Remark 7.1. We note the last property. Indeed, if (5.5) is a Hausdorff TS for which a compactifier exists, then (5.11) is a compactum. Now, we consider the scheme of the corresponding proof. 
So, as previously, we consider Hausdorff space (5.5). Therefore, (5.11) is a Hausdorff space also. We consider the case when some compactifier $(\mathbb{K}, \tilde{\tau}, \mu, v)$ exists. By Proposition 5.2 we obtain that

$$
\left(\operatorname{cl}\left(\mu^{1}(E), \tilde{\tau}\right),\left.\tilde{\tau}\right|_{\mathrm{cl}\left(\mu^{1}(E), \tilde{\tau}\right)}, \mu,\left(v \mid \operatorname{cl}\left(\mu^{1}(E), \tilde{\tau}\right)\right)\right)
$$

is a dense compactifier. Now, we suppose that

$$
\begin{aligned}
& Y \triangleq \operatorname{cl}\left(\mu^{1}(E), \tilde{\tau}\right),\left.\tau \triangleq \tilde{\tau}\right|_{\mathrm{cl}\left(\mu^{1}(E), \tilde{\tau}\right)}=\left.\tilde{\tau}\right|_{Y}, m \triangleq \mu, \\
& g \triangleq\left(v \mid \operatorname{cl}\left(\mu^{1}(E), \tilde{\tau}\right)\right)=(v \mid Y)
\end{aligned}
$$

By (7.12) we rename compactifier (7.11). So, $(Y, \tau, m, g)$ is our dense compactifier. Later, we apply procedure of the present section; as a result, we obtain that $(\tilde{\mathbf{H}}, \tilde{\mathbf{t}})$ is a compactum.

\section{Extension in Class of Ultrafilters of $\pi$-Systems}

Now, we consider a special variant of general construction of Sections 5-7. But, at first, we introduce some new notions (see [4] [8] [10] [14] [15] [16] [17] [18]). We fix $\mathcal{L} \in \pi[E]$ and consider the set

$$
\begin{aligned}
\mathbb{F}_{\lim }[E ; \mathcal{L} ; \mathbf{H} ; \mathbf{t}] & \triangleq\left\{f \in \mathbf{H}^{E} \mid \forall \mathcal{U} \in \mathbb{F}_{0}^{*}(\mathcal{L}) \exists h \in \mathbf{H}: f^{1}[\mathcal{U}] \stackrel{\mathbf{t}}{\Rightarrow} h\right\} \\
& =\left\{f \in \mathbf{H}^{E} \mid \forall \mathcal{U} \in \mathbb{F}_{0}^{*}(\mathcal{L}) \exists ! h \in \mathbf{H}: f^{1}[\mathcal{U}] \stackrel{\mathbf{t}}{\Rightarrow} h\right\} \\
& \in \mathcal{P}^{\prime}\left(\mathbf{H}^{E}\right)
\end{aligned}
$$

(we apply separability of TS (5.5); all mappings-constants with values in $\mathbf{H}$ (and defined on $E$ ) are elements of the set (8.1)). From (8.1), the next definition follows; namely, if $f \in \mathbb{F}_{\text {lim }}[E ; \mathcal{L} ; \mathbf{H} ; \mathbf{t}]$, then

$$
\varphi_{\lim }[f]: \mathbb{F}_{0}^{*}(\mathcal{L}) \rightarrow \mathbf{H}
$$

is defined by the following condition: under $\mathcal{U} \in \mathbb{F}_{0}^{*}(\mathcal{L})$, the point $\varphi_{\lim }[f](\mathcal{U}) \in \mathbf{H}$ has the property

$$
f^{1}[\mathcal{U}] \stackrel{\mathrm{t}}{\Rightarrow} \varphi_{\lim }[f](\mathcal{U})
$$

This corresponds to similar definition of $₫ 5$ in [10]. Of course, by (8.3)

$$
\begin{aligned}
& \varphi_{\lim }[f]^{1}(\mathbb{S})=\left\{h \in \mathbf{H} \mid \exists \mathcal{U} \in \mathbb{S}: f^{1}[\mathcal{U}] \stackrel{\mathrm{t}}{\Rightarrow} h\right\} \\
& \forall f \in \mathbb{F}_{\lim }[E ; \mathcal{L} ; \mathbf{H} ; \mathbf{t}] \forall \mathbb{S} \in \mathcal{P}\left(\mathbb{F}_{0}^{*}(\mathcal{L})\right)
\end{aligned}
$$

In connection with (4), we note statements in formula (5.4) of [10]; namely,

$$
\begin{aligned}
& \varphi_{\lim }[f]^{1}\left(\mathbb{F}_{0}^{*}(\mathcal{L} \mid \mathcal{E})\right) \subset(\mathbf{a s})[E ; \mathbf{H} ; \mathbf{t} ; f ; \mathcal{E}] \\
& \forall f \in \mathbb{F}_{\lim }[E ; \mathcal{L} ; \mathbf{H} ; \mathbf{t}] \forall \mathcal{E} \in \mathcal{P}^{\prime}(\mathcal{L})
\end{aligned}
$$

So, in (8.5), we obtain analog of (5.2). We recall that TS $(X, \tau)$ is called a regular space (regular TS) in the case when $(X, \tau)$ is $T_{1}$-space for which 
closed (in $(X, \tau))$ neighborhoods of every point $x \in X$ realize in totality a local base of $(X, \tau)$ for this point $X$. In this connection, we recall Formula (5.5) of [7]: if $(\mathbf{H}, \mathbf{t})$ is a regular TS, then

$$
\varphi_{\lim }[f] \in C\left(\mathbb{F}_{0}^{*}(\mathcal{L}), \mathbf{T}_{\mathcal{L}}^{*}[E], \mathbf{H}, \mathbf{t}\right) \forall f \in \mathbb{F}_{\lim }[E ; \mathcal{L} ; \mathbf{H} ; \mathbf{t}]
$$

Until end of this section, we suppose that $(\mathbf{H}, \mathbf{t})$ is a regular TS and $\mathcal{L} \in \tilde{\pi}^{0}[E]$. Moreover, now, we suppose that

$$
\mathbf{h} \in \mathbb{F}_{\lim }[E ; \mathcal{L} ; \mathbf{H} ; \mathbf{t}]
$$

Then, $\varphi_{\lim }[\mathbf{h}] \in \mathbf{H}^{\mathbb{F}_{0}^{*}(\mathcal{L})}$, and we obtain the natural concrete variant of (8.3)-(8.5); in addition, for $((E, \mathcal{L})-$ ult $)[\cdot] \triangleq((E, \mathcal{L})-$ ult $)[x]_{x \in E} \in \mathbb{F}_{0}^{*}(\mathcal{L})^{E}$

$$
\mathbf{h}=\varphi_{\lim }[\mathbf{h}] \circ((E, \mathcal{L})-\text { ult })[\cdot]
$$

As an addition to (8.5), we note (see (8.6)) the property noted in Proposition 4 of [10]:

$$
\varphi_{\lim }[f]^{1}\left(\mathbf{F}_{0}^{*}(\mathcal{L} \mid \mathcal{E})\right) \subset(\mathbf{a s})[E ; \mathbf{H} ; \mathbf{t} ; \mathbf{h} ; \mathcal{E}] \forall \mathcal{E} \in \beta[E]
$$

(we exploit (4.2), (5.2), (8.6), and (8.7)). As a corollary, from (8.9), we obtain that

$$
\varphi_{\lim }[f]^{1}\left(\mathbf{F}_{0}^{*}\left(\mathcal{L} \mid\left\{\bigcap_{\mathbf{f}_{\mathbf{f}}}(\mathcal{E})\right)\right) \subset(\mathbf{a s})[E ; \mathbf{H} ; \mathbf{t} ; \mathbf{h} ; \mathcal{E}] \forall \mathcal{E} \in \mathcal{P}^{\prime}(\mathcal{P}(E))\right.
$$

(we apply (4.1) and (4.2); indeed, under $\mathcal{S} \in \mathcal{P}^{\prime}(\mathcal{P}(E)$ ), the property $\{\cap\}_{\mathbf{f}}(\mathcal{E}) \in \beta[E]$ follows). It is useful to apply Remark 3 of [10].

In connection with (5.2), (8.7), and (8.10), we recall Proposition 3 of [10]:

$$
\text { (as) }\left[E ; \mathbb{F}_{0}^{*}(\mathcal{L}) ; \mathbf{T}_{\mathcal{L}}^{*}[E] ;((E, \mathcal{L})-\text { ult })[\cdot] ; \mathfrak{B}\right]=\mathbf{F}_{0}^{*}(\mathcal{L} \mid \mathfrak{B}) \forall \mathfrak{B} \in \beta[E]
$$

Attraction sets and compactifiers. We recall that the case of regular space $(\mathbf{H}, \mathbf{t})$ and $\mathcal{L} \in \tilde{\pi}^{0}[E]$ is considered (in this case TS $(\tilde{\mathbf{H}}, \tilde{\mathbf{t}})$ (5.11) is regular also). Moreover, in our case (8.7) is fulfilled. Finally, we suppose until end of this section that TS

$$
\left(\mathbb{F}_{0}^{*}(\mathcal{L}) ; \mathbf{T}_{\mathcal{L}}^{*}[E]\right)
$$

is compact. Then TS (8.12) is a zero-dimensional compactum. Therefore, from (3.7), (8.6), and (8.10), we obtain that

$$
\left(\mathbb{F}_{0}^{*}(\mathcal{L}), \mathbf{T}_{\mathcal{L}}^{*}[E],((E, \mathcal{L})-\text { ult })[\cdot], \varphi_{\lim }[\mathbf{h}]\right)
$$

is a dense compactifier. As a corollary, by (5.7) and (8.11)

$$
\begin{aligned}
(\mathbf{a s})[E ; \mathbf{H} ; \mathbf{t} ; \mathbf{h} ; \mathcal{E}] & =(\mathbf{a s})\left[E ; \mathbf{H} ; \mathbf{t} ; \mathbf{h} ;\{\cap\}_{\mathbf{f}}(\mathcal{E})\right] \\
& =\varphi_{\lim }[\mathbf{h}]^{1}\left(\mathbf{F}_{0}^{*}\left(\mathcal{L} \mid\{\cap\}_{\mathbf{f}}(\mathcal{E})\right)\right) \forall \mathcal{E} \in \mathcal{P}^{\prime}(\mathcal{P}(E))
\end{aligned}
$$

In connection with (8.14), we note the following important particular case:

$$
(\mathbf{a s})[E ; \mathbf{H} ; \mathbf{t} ; \mathbf{h} ; \mathfrak{B}]=\varphi_{\lim }[\mathbf{h}]^{1}\left(\mathbf{F}_{0}^{*}(\mathcal{L} \mid \mathfrak{B})\right) \forall \mathfrak{B} \in \beta[E]
$$

Of course, (8.15) is sufficient for majority of applications. From (8.4), (8.7), 
and (8.15), we obtain that

$$
\text { (as) }[E ; \mathbf{H} ; \mathbf{t} ; \mathbf{h} ; \mathfrak{B}]=\left\{z \in \mathbf{H} \mid \exists \mathcal{U} \in \mathbf{F}_{0}^{*}(\mathcal{L} \mid \mathfrak{B}): \mathbf{h}^{1}[\mathcal{U}] \stackrel{\mathbf{t}}{\Rightarrow} z\right\} \forall \mathfrak{B} \in \beta[E]
$$

It is useful to note that by (8.15) and (8.16) (in our case) ultrafilters from $\mathbf{F}_{0}^{*}(\mathcal{L} \mid \mathfrak{B})$, where $\mathfrak{B} \in \beta[E]$, can be considered as analogs both generalized and approximate solutions of J. Warga (see constructions of ch.III of [2]).

Remark 8.1. The compactness property of TS (8.12) is very important (see (8.14)-(8.16)). Now, we note only Remark 3.1 (of course, more general cases are known: see $\$ 6$ in [14]). Another important question is connected with (8.7). More exactly, we obtain the serious question about representation of the set (8.1). Now, we discuss only two possibilities for $\mathbf{h}$.

1) Let $(\tilde{\mathbf{H}}, \tilde{\mathbf{t}})$ (5.11) be a compactum (in this connection, we note Remark 7.1). In addition, by (5.8) $\mathbf{h} \in \tilde{\mathbf{H}}^{E}$. We strive to obtain conditions sufficient for (8.7). For this, we apply the construction of $\$ 5$ in [14]. For any $h \in \tilde{\mathbf{H}}$, we introduce the family

$$
(h-\text { bas })[\tilde{\mathbf{t}}] \triangleq\left\{\mathcal{S} \in \mathcal{P}\left(N_{\tilde{\mathbf{t}}}(h)\right) \mid \forall A \in N_{\tilde{\mathbf{t}}}(h) \exists B \in \mathcal{S}: B \subset A\right\}
$$

of all local bases of TS $(\tilde{\mathbf{H}}, \tilde{\mathbf{t}})$ at the point $h$. In this item, we suppose that

$$
\forall h \in \tilde{\mathbf{H}} \exists \tilde{\mathcal{H}} \in(h-\text { bas })[\tilde{\mathbf{t}}]: \mathbf{h}^{-1}(H) \in \mathcal{L} \forall H \in \tilde{\mathcal{H}}
$$

So, (8.17) is a condition of the "local measurability" of $\mathbf{h}$. We recall about compactness of TS (5.11). Then, by Corollary 5.1 of [14]

$$
\forall \mathcal{U} \in \mathbb{F}_{0}^{*}(\mathcal{L}) \exists ! h \in \tilde{\mathbf{H}}: \mathbf{h}^{1}[\mathcal{U}] \stackrel{\tilde{\mathbf{t}}}{\Rightarrow} h
$$

Let $\mathfrak{U} \in \mathbb{F}_{0}^{*}(\mathcal{L})$ and $\tilde{h} \in \tilde{\mathbf{H}}$ be a point such that (see (8.18))

$$
\mathbf{h}^{1}[\mathfrak{U}] \stackrel{\tilde{\mathbf{t}}}{\Rightarrow} \tilde{h}
$$

In addition, $\mathbf{h}^{1}[\mathfrak{U}] \in \beta_{0}[\tilde{\mathbf{H}}]$. By (3.1) and (8.19)

$$
N_{\tilde{\mathbf{t}}}(\tilde{h}) \subset(\tilde{\mathbf{H}}-\mathbf{f i})\left[\mathbf{h}^{1}[\mathfrak{U}]\right]
$$

We recall that (see Formula (2.3.8) of [11]) by (5.10)

$$
N_{\tilde{\mathbf{t}}}(\tilde{h})=\left.N_{\mathbf{t}}(\tilde{h})\right|_{\tilde{\mathbf{H}}}=\left\{S \cap \tilde{\mathbf{H}}: S \in N_{\mathbf{t}}(\tilde{h})\right\}
$$

We recall that

$$
\begin{aligned}
(\tilde{\mathbf{H}}-\mathbf{f i})\left[\mathbf{h}^{1}[\mathfrak{U}]\right] & =\left\{T \in \mathcal{P}(\tilde{\mathbf{H}}) \mid \exists B \in \mathbf{h}^{1}[\mathfrak{U}]: B \subset T\right\} \\
& \subset\left\{T \in \mathcal{P}(\mathbf{H}) \mid \exists B \in \mathbf{h}^{1}[\mathfrak{U}]: B \subset T\right\}
\end{aligned}
$$

In addition, by the choice of $\mathbf{h}$ we obtain that $\mathbf{h}^{1}[\mathfrak{U}] \in \beta_{0}[\mathbf{H}]$. So, the filter

$$
(\mathbf{H}-\mathbf{f i})\left[\mathbf{h}^{1}[\mathfrak{U}]\right]=\left\{T \in \mathcal{P}(\mathbf{H}) \mid \exists B \in \mathbf{h}^{1}[\mathfrak{U}]: B \subset T\right\} \in \mathfrak{F}[\mathbf{H}]
$$

is defined; by (8.22) the inclusion 


$$
(\tilde{\mathbf{H}}-\mathbf{f i})\left[\mathbf{h}^{1}[\mathfrak{U}]\right] \subset(\mathbf{H}-\mathbf{f i})\left[\mathbf{h}^{1}[\mathfrak{U}]\right]
$$

follows. By (8.20) and (8.23) the next inclusion

$$
N_{\tilde{\mathbf{t}}}(\tilde{h}) \subset(\mathbf{H}-\mathbf{f i})\left[\mathbf{h}^{1}[\mathfrak{U}]\right]
$$

is realized. Now, we exploit (2.5) and (8.21). Indeed, let $\mathbb{S} \in N_{\mathbf{t}}(\tilde{h})$. Then, by (8.21) $\mathbb{S} \cap \tilde{\mathbf{H}} \in N_{\tilde{\mathbf{t}}}(\tilde{h})$. By (8.24) the inclusion $\mathbb{S} \cap \tilde{\mathbf{H}} \in(\mathbf{H}-\mathbf{f i})\left[\mathbf{h}^{1}[\mathfrak{U}]\right]$ follows, where $\mathbb{S} \cap \tilde{\mathbf{H}} \subset \mathbb{S}$. Since $\mathbb{S} \in \mathcal{P}(\mathbf{H})$, by (2.5) $\mathbb{S} \in(\mathbf{H}-\mathbf{f i})\left[\mathbf{h}^{1}[\mathfrak{U}]\right]$. So, we obtain that $N_{\mathbf{t}}(\tilde{h}) \subset(\mathbf{H}-\mathbf{f i})\left[\mathbf{h}^{1}[\mathfrak{U}]\right]$. As a corollary,

$$
\mathbf{h}^{1}[\mathfrak{U}] \stackrel{\mathbf{t}}{\Rightarrow} \tilde{h}
$$

where $\tilde{h} \in \tilde{\mathbf{H}}$. In particular, $\tilde{h} \in \mathbf{H}$. Since the choice of $\mathfrak{U}$ was arbitrary, it is established that

$$
\forall \mathcal{U} \in \mathbb{F}_{0}^{*}(\mathcal{L}) \exists h \in \mathbf{H}: \mathbf{h}^{1}[\mathcal{U}] \stackrel{\mathbf{t}}{\Rightarrow} h
$$

From (8.1), we obtain that, in our case, (8.7) is fulfilled. So, (8.17) and compactness of TS (5.11) are sufficient for (8.7).

2) Now, we consider the variant connected with application of uniform limits of real-valued step-functions (the more general construction is given in [15]). In this item, we suppose that $\mathbf{H}=\mathbb{R}^{\Gamma}$, where $\Gamma$ is a nonempty set. So, elements of $\mathbf{H}$ are functionals on $\Gamma$ and only they. We apply $\mathbf{h} \in \mathbf{H}^{E}$ and suppose that

$$
\mathbf{h}(\cdot)(\gamma) \triangleq(\mathbf{h}(x)(\gamma))_{x \in E} \in \mathbb{R}^{E} \forall \gamma \in \Gamma
$$

In (8.25), we obtain real-valued components of $\mathbf{h}$. We recall that $E$ is equipped with $\mathcal{L} \in \pi[E]$. Now, we suppose that $\mathcal{L}$ is a semialgebra of subsets of $E$ (of course, the variant of an algebra of subsets of $E$ is possible also). Under $\Sigma \in \mathcal{P}(E)$, by $\chi_{\Sigma}$ we denote indicator of the set $\Sigma: \chi_{\Sigma} \in \mathbb{R}^{E}, \chi_{\Sigma}(x) \triangleq 0$ under $x \in E \backslash \Sigma$, and $\chi_{\Sigma}(y) \triangleq 1$ under $y \in \Sigma$. By $B_{0}(E, \mathcal{L})$ we denote the linear span of the set $\left\{\chi_{L}: L \in \mathcal{L}\right\}$ (we apply the pointwise linear operations in $\mathbb{R}^{E}$ ) obtaining a subset of the set $\mathbb{B}(E)$ of all bounded real-valued functions on $E$. We equip the linear space $\mathbb{B}(E)$ with traditional sup-norm $\|\cdot\|$ (see ch.IV, Section 2, item 13 of [16]). Then, $B_{0}(E, \mathcal{L}) \subset \mathbb{B}(E)$. The closure of $B_{0}(E, \mathcal{L})$ in $\mathbb{B}(E)$ with topology generated by norm $\|\cdot\|$ is denoted by $B(E, \mathcal{L})$ (if $\mathcal{L} \in(\operatorname{alg})[E]$, then $B(E, \mathcal{L})$ corresponds to ch.IV, Section 5 of [16]). In particular, $B(E, \mathcal{L})$ with norm induced from $(\mathbb{B}(E),\|\cdot\|)$ is a Banach space.

Suppose that $\mathbf{h}(\cdot)(\gamma) \in B(E, \mathcal{L}) \forall \gamma \in \Gamma$. Now, we define a variant of topology t . Namely, we suppose that $\mathbf{t}=\otimes^{\Gamma}\left(\tau_{\mathbb{R}}\right)$ is the natural topology of the Tychonoff power of $\mathbb{R}$ with usual $|\cdot|$-topology $\tau_{\mathbb{R}}$ for the case when $\Gamma$ is the indexed set. So, we consider the Tichonoff product of samples $\left(\mathbb{R}, \tau_{\mathbb{R}}\right)$ under indexed set $\Gamma$. Using Proposition 6.3 of [15], we obtain that (8.7) is fulfilled (in [15], the concrete variant of the mapping $\varphi_{\lim }[\mathbf{h}]$ is indicated; also, we note Remark 6.2 of [15]). 
So, under some natural conditions, the property (8.7) is realized.

\section{Some Topological Properties of Attraction Sets in Space of Ultrafilters of $\pi$-Systems}

In this section, we fix $\mathcal{L} \in \pi[E]$ and consider the set $\mathbb{F}_{0}^{*}(\mathcal{L})$ (see Section 2). In this construction, we follow to [17].

Proposition 9.1. If $L_{1} \in \mathcal{L}$ and $L_{2} \in \mathcal{L}$, then

$$
\left(L_{1} \subset L_{2}\right) \Rightarrow\left(\Phi_{\mathcal{L}}\left(L_{1}\right) \subset \Phi_{\mathcal{L}}\left(L_{2}\right)\right)
$$

in addition, if $\mathcal{L} \in \tilde{\pi}^{0}[E]$, then $\forall \Lambda_{1} \in \mathcal{L} \forall \Lambda_{2} \in \mathcal{L}$

$$
\left(\Phi_{\mathcal{L}}\left(\Lambda_{1}\right) \subset \Phi_{\mathcal{L}}\left(\Lambda_{2}\right)\right) \Rightarrow\left(\Lambda_{1} \subset \Lambda_{2}\right)
$$

The corresponding proof is given in $\$ 4$ of article [17]. In connection with Proposition 9.1, we suppose until end of this section that $\mathcal{L} \in \tilde{\pi}^{0}[E]$. Then, by Proposition $9.1 \forall L_{1} \in \mathcal{L} \forall L_{2} \in \mathcal{L}$

$$
\left(L_{1} \subset L_{2}\right) \Leftrightarrow\left(\Phi_{\mathcal{L}}\left(L_{1}\right) \subset \Phi_{\mathcal{L}}\left(L_{2}\right)\right)
$$

As a corollary, in our case, the mapping

$$
L \mapsto \Phi_{\mathcal{L}}(L): \mathcal{L} \rightarrow(\mathbb{U F})[E ; \mathcal{L}]
$$

is a bijection from $\mathcal{L}$ onto $(\mathbb{U F})[E ; \mathcal{L}]$.

We fix $\mathcal{E} \in \mathcal{P}^{\prime}(\mathcal{L})$ until end of this section (we apply $\mathcal{E}$ as the family defining constraints of asymptotic character; in particular, $\mathcal{E} \in \mathcal{P}^{\prime}(\mathcal{P}(E))$. In this and next sections, it is supposed that

$$
\Sigma_{0} \triangleq \bigcap_{\Sigma \in \mathcal{E}} \Sigma \in \mathcal{L}
$$

(in $\$ 6$ of [17], the examples of $(E, \mathcal{L})$ and $\mathcal{E}$ with (9.2) are given; we note that (9.2) is fulfilled under

$$
\bigcap_{\Sigma \in \mathcal{E}} \Sigma=\varnothing
$$

the last case is considered in [18] specially). By (2.11) and (9.2) the set

$$
\begin{aligned}
\Phi_{\mathcal{L}}\left(\Sigma_{0}\right) & =\left\{\mathcal{U} \in \mathbb{F}_{0}^{*}(\mathcal{L}) \mid \Sigma_{0} \in \mathcal{U}\right\}=\left\{\mathcal{U} \in \mathbb{F}_{0}^{*}(\mathcal{L}) \mid \Sigma_{0} \cap U \neq \varnothing \forall U \in \mathcal{U}\right\} \\
& \in \mathbf{T}_{\mathcal{L}}^{*}[E] \cap \mathbf{C}_{\mathbb{F}_{0}^{*}(\mathcal{L})}\left[\mathbf{T}_{\mathcal{L}}^{*}[E]\right]
\end{aligned}
$$

is defined. We recall that $\mathbb{F}_{0}^{*}(\mathcal{L} \mid \mathcal{E})$ coincides with intersection of all sets $\Phi_{\mathcal{L}}(\Sigma), \Sigma \in \mathcal{E}$. In Theorem 6.1 of [17], it is established that

$$
\left(\mathbf{T}_{\mathcal{L}}^{*}[E]-\operatorname{Int}\right)\left[\mathbb{F}_{0}^{*}(\mathcal{L} \mid \mathcal{E})\right]=\Phi_{\mathcal{L}}\left(\Sigma_{0}\right)
$$

In particular, from (9.3) and (9.4), the following property follows: the interior of $\mathbb{F}_{0}^{*}(\mathcal{L} \mid \mathcal{E})$ is an open-closed set.

Remark 9.1. Recall (8.11). Then, under $\mathcal{E} \in \beta[E]$ (for which $\mathcal{E} \subset \mathcal{L}$ and (9.2) is fulfilled), we obtain that

$$
\text { (as) }\left[E ; \mathbb{F}_{0}^{*}(\mathcal{L}) ; \mathbf{T}_{\mathcal{L}}^{*}[E] ;((E, \mathcal{L})-\text { ult })[\cdot] ; \mathcal{E}\right]=\mathbb{F}_{0}^{*}(\mathcal{L} \mid \mathcal{E})
$$

So, in this case, by (9.4) the interior of auxiliary AS is defined (see definitions 
of Section 2). Since $\mathbb{F}_{0}^{*}(\mathcal{L} \mid \mathcal{E}) \in \mathbf{C}_{\mathbb{F}_{0}^{*}(\mathcal{L})}\left[\mathbf{T}_{\mathcal{L}}^{*}[E]\right]$, we obtain that

$$
\left(\mathbf{T}_{\mathcal{L}}^{*}[E]-\operatorname{Fr}\right)\left[\mathbb{F}_{0}^{*}(\mathcal{L} \mid \mathcal{E})\right]=\mathbb{F}_{0}^{*}(\mathcal{L} \mid \mathcal{E}) \backslash \Phi_{\mathcal{L}}\left(\Sigma_{0}\right)
$$

(the frontier of the set (9.5) is realized). By (9.6) the next inclusion is realized

$$
\mathbb{F}_{0}^{*}(\mathcal{L} \mid \mathcal{E}) \backslash \Phi_{\mathcal{L}}\left(\Sigma_{0}\right) \subset \operatorname{cl}\left(\mathbb{F}_{0}^{*}(\mathcal{L}) \backslash \mathbb{F}_{0}^{*}(\mathcal{L} \mid \mathcal{E}), \mathbf{T}_{\mathcal{L}}^{*}[E]\right)
$$

Now, we consider questions connected with approximate realization of ultrafilters of $\mathbb{F}_{0}^{*}(\mathcal{L} \mid \mathcal{E}) \backslash \Phi_{\mathcal{L}}\left(\Sigma_{0}\right)$. So, we consider questions connected with approximate realizability of elements of (9.6). Then

$$
\left(\mathbf{T}_{\mathcal{L}}^{*}[E]-\mathrm{Fr}\right)\left[\mathbb{F}_{0}^{*}(\mathcal{L} \mid \mathcal{E})\right]=\mathbb{F}_{0}^{*}(\mathcal{L} \mid \mathcal{E}) \backslash \mathrm{cl}\left(((E, \mathcal{L})-\text { ult })[\cdot]^{1}\left(\Sigma_{0}\right), \mathbf{T}_{\mathcal{L}}^{*}[E]\right)
$$

So, ultrafilters of the frontier of $\mathbb{F}_{0}^{*}(\mathcal{L} \mid \mathcal{E})$ are not realizable "outwards". In addition, by (3.6) we obtain that

$$
\operatorname{cl}\left(((E, \mathcal{L})-\text { ult })[\cdot]^{1}\left(E \backslash \Sigma_{0}\right), \mathbf{T}_{\mathcal{L}}^{*}[E]\right)=\mathbf{F}_{0}^{*}\left[\mathcal{L} \mid E \backslash \Sigma_{0}\right]
$$

Moreover, by (9.6) we obtain the following

Proposition 9.1. The following inclusion takes place:

$$
\left(\mathbf{T}_{\mathcal{L}}^{*}[E]-\operatorname{Fr}\right)\left[\mathbb{F}_{0}^{*}(\mathcal{L} \mid \mathcal{E})\right] \subset \mathbf{F}_{0}^{*}\left[\mathcal{L} \mid E \backslash \Sigma_{0}\right]
$$

Proof. By (3.6), (3.7), and additivity of the closure we obtain that

$$
\begin{aligned}
\mathbb{F}_{0}^{*}(\mathcal{L}) & =\operatorname{cl}\left(\mathbb{F}_{0, \mathrm{t}}^{*}(\mathcal{L}), \mathbf{T}_{\mathcal{L}}^{*}[E]\right)=\operatorname{cl}\left(((E, \mathcal{L})-\text { ult })[\cdot]^{1}(E), \mathbf{T}_{\mathcal{L}}^{*}[E]\right) \\
& =\operatorname{cl}\left(((E, \mathcal{L})-\text { ult })[\cdot]^{1}\left(\Sigma_{0} \cup\left(E \backslash \Sigma_{0}\right)\right), \mathbf{T}_{\mathcal{L}}^{*}[E]\right) \\
& =\operatorname{cl}\left(((E, \mathcal{L})-\text { ult })[\cdot]^{1}\left(\Sigma_{0}\right) \cup((E, \mathcal{L})-\text { ult })[\cdot]^{1}\left(E \backslash \Sigma_{0}\right), \mathbf{T}_{\mathcal{L}}^{*}[E]\right) \\
& =\operatorname{cl}\left(((E, \mathcal{L})-\text { ult })[\cdot]^{1}\left(\Sigma_{0}\right), \mathbf{T}_{\mathcal{L}}^{*}[E]\right) \cup \operatorname{cl}\left(((E, \mathcal{L})-\text { ult })[\cdot]^{1}\left(E \backslash \Sigma_{0}\right), \mathbf{T}_{\mathcal{L}}^{*}[E]\right) \\
& =\Phi_{\mathcal{L}}\left(\Sigma_{0}\right) \cup \mathbf{F}_{0}^{*}\left[\mathcal{L} \mid E \backslash \Sigma_{0}\right]
\end{aligned}
$$

Since $\mathbb{F}_{0}^{*}(\mathcal{L} \mid \mathcal{E}) \subset \mathbb{F}_{0}^{*}(\mathcal{L})$, by (9.6) we obtain the required inclusion (9.9).

Corollary 9.1. The next inclusion is realized:

$$
\begin{aligned}
& \left(\mathbf{T}_{\mathcal{L}}^{*}[E]-\operatorname{Fr}\right)\left[\mathbb{F}_{0}^{*}(\mathcal{L} \mid \mathcal{E})\right] \\
& \subset \operatorname{cl}\left(((E, \mathcal{L})-\text { ult })[\cdot]^{1}\left(E \backslash \Sigma_{0}\right), \mathbf{T}_{\mathcal{L}}^{*}[E]\right) \backslash \operatorname{cl}\left(((E, \mathcal{L})-\text { ult })[\cdot]^{1}\left(\Sigma_{0}\right), \mathbf{T}_{\mathcal{L}}^{*}[E]\right)
\end{aligned}
$$

The corresponding proof is reduced to immediate combination of (9.7), (9.8), and Proposition 9.1. So, ultrafilters of the frontier of $\mathbb{F}_{0}^{*}(\mathcal{L} \mid \mathcal{E})$ assume approximate realization only by points of the set $E \backslash \Sigma_{0}$.

We recall that by (9.2) and (9.4)

$$
\left(\mathbf{T}_{\mathcal{L}}^{*}[E]-\text { Int }\right)\left[\mathbb{F}_{0}^{*}(\mathcal{L} \mid \mathcal{E})\right] \in \mathbf{T}_{\mathcal{L}}^{*}[E] \cap \mathbf{C}_{\mathbb{F}_{0}^{*}(\mathcal{L})}\left[\mathbf{T}_{\mathcal{L}}^{*}[E]\right]
$$

Remark 9.2. If $\left(\mathbf{T}_{\mathcal{L}}^{*}[E]-\operatorname{Fr}\right)\left[\mathbb{F}_{0}^{*}(\mathcal{L} \mid \mathcal{E})\right] \neq \varnothing$, then

$$
\mathbb{F}_{0}^{*}(\mathcal{L} \mid \mathcal{E}) \in \mathbf{C}_{\mathbb{F}_{0}^{*}(\mathcal{L})}\left[\mathbf{T}_{\mathcal{L}}^{*}[E]\right] \backslash(\text { can }- \text { clos })\left[\mathbf{T}_{\mathcal{L}}^{*}[E]\right]
$$

Indeed, let $\left(\mathbf{T}_{\mathcal{L}}^{*}[E]-\mathrm{Fr}\right)\left[\mathbb{F}_{0}^{*}(\mathcal{L} \mid \mathcal{E})\right] \neq \varnothing$. By $(9.10)$ 


$$
\operatorname{cl}\left(\left(\mathbf{T}_{\mathcal{L}}^{*}[E]-\operatorname{Int}\right)\left[\mathbb{F}_{0}^{*}(\mathcal{L} \mid \mathcal{E})\right], \mathbf{T}_{\mathcal{L}}^{*}[E]\right)=\left(\mathbf{T}_{\mathcal{L}}^{*}[E]-\operatorname{Int}\right)\left[\mathbb{F}_{0}^{*}(\mathcal{L} \mid \mathcal{E})\right]
$$

Using (9.4) and (9.6), we obtain (under our condition) that

$$
\mathbb{F}_{0}^{*}(\mathcal{L} \mid \mathcal{E}) \neq \operatorname{cl}\left[\left(\mathbf{T}_{\mathcal{L}}^{*}[E]-\operatorname{Int}\right)\left[\mathbb{F}_{0}^{*}(\mathcal{L} \mid \mathcal{E})\right], \mathbf{T}_{\mathcal{L}}^{*}[E]\right]
$$

So, $\mathbb{F}_{0}^{*}(\mathcal{L} \mid \mathcal{E}) \notin($ can - clos $)\left[\mathbf{T}_{\mathcal{L}}^{*}[E]\right]$.

\section{Some Additions}

We follow to constructions of Section 4 of article [8] under consideration of the case $\mathcal{L} \in \pi_{0}^{\prime}[E]$, where $E$ is a nonempty set. In this case, the open-closed sets $\Phi_{\mathcal{L}}(\{x\}), x \in E$ are defined. In addition, under $x \in E$

$$
\Phi_{\mathcal{L}}(\{x\})=\{((E, \mathcal{L})-\text { ult })[x]\}
$$

As a corollary, we obtain the following equality

$$
\left(\mathbf{T}_{\mathcal{L}}^{*}[E]-\text { isol }\right)\left[\mathbb{F}_{0}^{*}(\mathcal{L})\right]=\mathbb{F}_{0, \mathbf{t}}^{*}(\mathcal{L})
$$

therefore (see Section 3) we obtain that

$$
\left(\mathbf{T}_{\mathcal{L}}^{*}[E]-\text { isol }\right)\left[\mathbb{F}_{0}^{*}(\mathcal{L})\right] \in\left(\mathbf{T}_{\mathcal{L}}^{*}[E]-\text { dens }\right)\left[\mathbb{F}_{0}^{*}(\mathcal{L})\right]
$$

So, in our case, TS of type (3.3) has everywhere dense subset consisting from isolated points. These points are trivial ultrafilters.

From (10.1) and (10.2), we obtain ( under $\mathcal{L} \in \pi_{0}^{\prime}[E]$ ) that

$$
((E, \mathcal{L})-\text { ult })[\cdot]^{1}(A)=\{((E, \mathcal{L})-\text { ult })[x]: x \in A\} \in \mathbf{T}_{\mathcal{L}}^{*}[E] \forall A \in \mathcal{P}(E)
$$

From (3.6) and (10.3), the following important property (see p. 222 in [8]) is realized:

$$
\mathbf{F}_{0}^{*}[\mathcal{L} \mid A] \in(\operatorname{can}-\operatorname{clos})\left[\mathbf{T}_{\mathcal{L}}^{*}[E]\right] \forall A \in \mathcal{P}(E)
$$

Until end of this section, we fix a family $\mathcal{E} \in \mathcal{P}^{\prime}(\mathcal{L})$.

Proposition 10.1. Let (9.2) holds and $\mathbb{F}_{0}^{*}(\mathcal{L} \mid \mathcal{E}) \backslash \Phi_{\mathcal{L}}\left(\Sigma_{0}\right) \neq \varnothing$. Then

$$
\mathbb{F}_{0}^{*}(\mathcal{L} \mid \mathcal{E}) \neq \mathbf{F}_{0}^{*}[\mathcal{L} \mid A] \forall A \in \mathcal{P}(E)
$$

Proof. By (9.6) we have that $\left(\mathbf{T}_{\mathcal{L}}^{*}[E]-\mathrm{Fr}\right)\left[\mathbb{F}_{0}^{*}(\mathcal{L} \mid \mathcal{E})\right] \neq \varnothing$. Then, from Remark 9.2, we obtain the property

$$
\mathbb{F}_{0}^{*}(\mathcal{L} \mid \mathcal{E}) \notin(\operatorname{can}-\operatorname{clos})\left[\mathbf{T}_{\mathcal{L}}^{*}[E]\right]
$$

By (10.4) we obtain the required statement.

As a corollary, the following property is realized: if the conditions of Proposition 10.1 are fulfilled, then by (3.6)

$$
\mathbb{F}_{0}^{*}(\mathcal{L} \mid \mathcal{E}) \neq \operatorname{cl}\left(((E, \mathcal{L})-\text { ult })[\cdot]^{1}(A), \mathbf{T}_{\mathcal{L}}^{*}[E]\right) \forall A \in \mathcal{P}(E)
$$

Now, we discuss (10.5). The expression on the right-hand side of (10.5) corresponds to "standard" realization of generalized elements. Namely, we have some set $A, A \subset E$ of usual solutions. These solutions are transformed in generalized elements (here, in ultrafilters). The obtained set of ultrafilters is transformed to a closure (we obtain $\mathbf{F}_{0}^{*}[\mathcal{L} \mid A]$ ). By (10.5) and Proposition 10.1 
we no obtain $\mathbb{F}_{0}^{*}(\mathcal{L} \mid \mathcal{E})$ under every $A, A \subset E$ (of course, we keep in mind that (9.2) and conditions of Proposition 10.1 are fulfilled and the sets-closures on the right-hand side of (10.5) are used). So, auxiliary AS (see (8.11) and Section 2) one cannot to obtain by closure of some subset of the initial set $E$.

\section{Supported}

This work was supported by Russian Foundation for Basic Research (project no.15-01-07909).

\section{References}

[1] Krasovskii, N.N. (1968) The Theory of the Control of Motion. Nauka, Moscow. (In Russian)

[2] Warga, J. (1977) Optimal Control of Differential and Functional Equations. Academic Press, New York.

[3] Chentsov, A.G. (1997) Asymptotic Attainability. Kluwer Academic Publishing, Dordrecht. https://doi.org/10.1007/978-94-017-0805-0

[4] Chentsov, A.G. (2016) Compactifiers in Extension Constructions for Reachability Problems with Constraints of Asymptotic Nature. Trudy Instituta Matematiki $i$ Mekhaniki UrO RAN, 22, 1.

[5] Bulinskii, A.V. and Shiryaev, A.N. (2005) Theory of Stochastic Processes. Classical University Textbook, Fizmatlit, Moscow. (In Russian)

[6] Bourbaki, N. (1995) General Topology. Springer-Verlag, Berlin Heidelberg.

[7] Chentsov, A.G. (2011) Filters and Ultrafilters in the Constructions of Attraction Sets. Vestn. Udmurtsk. Univ. Mat. Mekh. Komp. Nauki, No. 1.

[8] Chentsov, A.G. (2015) To Question about Realization of Attraction Elements in Abstract Attainability Problems. Vestn. Udmurtsk. Univ. Mat. Mekh. Komp. Nauki, 25,2 .

[9] Chentsov, A.G. (2011) On One Example of Representing the Ultrafilter Space for an Algebra of Sets. Trudy Instituta Matematiki i Mekhaniki UrO RAN, 17, 4.

[10] Chentsov, A.G. (2014) Some Ultrafilter Properties Connected with Extension Constructions. Vestn. Udmurtsk. Univ. Mat. Mekh. Komp. Nauki, No. 1.

[11] Chentsov, A.G. and Morina, S.I. (2002) Extensions and Relaxations. Kluwer Acad. Publ., Dordrecht, Boston, London. https://doi.org/10.1007/978-94-017-1527-0

[12] Engelking, R. (1977) General Topology. PWN, Warsaw.

[13] Kelley, J.L. (1975) General Topology. Springer, New York.

[14] Chentsov, A.G. (2012) The Transformation of Ultrafilters and Their Application in Constructions of Attraction Sets. Vestn. Udmurtsk. Univ. Mat. Mekh. Komp. Nauki, No. 3.

[15] Chentsov, A.G. (2012) Tier Mappings and Ultrafilter-Based Transformations. Trudy Instituta Matematiki i Mekhaniki UrO RAN, 18, 4.

[16] Dunford, N. and Schwartz, J.T. (1988) Linear Operators, Part 1: General Theory.

[17] Chentsov, A.G. (2014) To the Validity of Constraints in the Class of Generalized Elements. Vestn. Udmurtsk. Univ. Mat. Mekh. Komp. Nauki, No. 3.

[18] Chentsov, A.G. (2015) An Abstract Reachability Problem: Purely Asymptotic? Version, Trudy Instituta Matematiki i Mekhaniki UrO RAN, 21, 2. 
Submit or recommend next manuscript to SCIRP and we will provide best service for you:

Accepting pre-submission inquiries through Email, Facebook, LinkedIn, Twitter, etc. A wide selection of journals (inclusive of 9 subjects, more than 200 journals)

Providing 24-hour high-quality service

User-friendly online submission system

Fair and swift peer-review system

Efficient typesetting and proofreading procedure

Display of the result of downloads and visits, as well as the number of cited articles Maximum dissemination of your research work

Submit your manuscript at: http://papersubmission.scirp.org/

Or contact iim@scirp.org 